\title{
DAS NEUSTE PROBLEM DES KLASSENKAMPFS - DER KAMPF GEGEN DIE ABBILDTHEORIE
}

\author{
Arnhelm Neusüss / Frank Unger
}

\section{Inhalt:}

I. Die Kritik der Kritiker, S. 263 (1. Die Quelle, S. 264; 2. Die Objektivität der Widerspiegelung und der Gegenstand der Widerspiegelungstheorie, S. 266; 3. Die Natur der Anpassung und die Anpassung der Natur, S. 276; 4. Die Wahrheit der ,relativen Wahrheit", S. 281), II. Die Erkenntnistheorie der Kritiker, S. 284 (1. Geschichtsbild und Erwartung an Erkenntnistheorie, S. $285 ; 2$. Erkenntnistheorie und materialistische Wissenschaft, S. 288; 3. Revolution der Erkenntnistheorie, S. 290)

Der in der Nummer 16 der „Probleme des Klassenkampfs“" vorgeführten endguiltigen Vernichtung der „Abbild- oder Widerspiegelungstheorie“ (1) möchten wir einige kritische Anmerkungen nachschicken. Vorab sei bemerkt: wir stimmen mit den Autoren darin überein, daß „Kritik an einer falschen Theorie im Marxismus kein Sakrileg" bedeuten kann, und würde dabei gar Kritik geübt ,,an den sozialistischen Staaten“. Selbst das Rütteln an einem ihrer ideologischen „Eckpfeiler", das diese Staaten erschüttern könnte, würden wir, wenn es der Wahrheitsfindung dient, so wenig scheuen wie die kritischen Autoren. Und ebenso wenig wären wir bereit, ein - bei wem auch immer - etwa ,,in den westlichen Ländern aufkeimendes Bedürfnis nach einer marxistischen Wissenschaftskritik" zu beschneiden, und wir würden, wenn denn solches Bedürfnis durch eine ,unkritisierte Abbild theorie ... in die Irre geleitet" wird, die dann dringende Kritik sogar, im Gegensatz zu den Kritikern, „leichten Herzens" vornehmen (2).

Denn auf die Gemütsverfassung kommt es beim Kritiküben weniger an als auf die Stichhaltigkeit, zumal bei einer Kritik mit derart unabsehbaren Folgen, sei es für abgestorbene Dialektik, sei es für keimende Bedürfnisse. Die hier vorgelegte Kritik an der Abbildtheorie entbehrt jedoch jeder Grundlage. Wir werden zeigen, daß das Verfahren der Kritiker mit Fälschung arbeitet - wie weit dies subjektiv bewußt war, oder ob hier ein - dann allerdings frappie render - Fall von selektiver Wahrnehmung vorliegt, können wir nicht entscheiden. Da es sich bei den Auslassungen der Kritiker nicht um Meinungen und Interpretationen handelt, die sich noch innerhalb des Bereichs wissenschaftlicher Textanalyse artikulieren, kann es sich auch für uns nicht darum handeln, diesen Auffassungen andere - unsere - Meinungen und Inter-

Bodo v. Greiff - Hanne Herkommer, Die Abbildtheorie und „Das Argument", in: Probleme des Klassenkampfs, 16, IV, Jhg. 1974: Nr. 4

2 Alle Zitate S. 163. Im folgenden setzen wir hinter Zitate aus dem PROKLA-Aufsatz die jeweilige Seitenzahl in Klammern in den Kontext. 
pretationen entgegenzuhalten. Es kommt vielmehr darauf an, im Aufweis der Haltlosigkeit der „Kritik“ allererst die Grundlage wiederherzustellen, auf der sich über Abbildtheorie sinnvoll und wissenschaftlich legitim diskutieren läßt: nämlich die Rekonstruktion ihrer authentischen Positionen, die unumgänglich wird (3), wenn deren Verfälschung aufzuweisen ist. Was an den abbildtheoretischen Auffassungen problematisch, kritisierbar und kritisierenswert sein mag, kann deshalb hier nicht unser Thema sein.

Wir verfolgen die eben skizzierte Aufgabe im Teil I unserer Anmerkungen (,Die Kritik der Kritiker"). In einem zweiten Teil („Die Erkenntnistheorie der Kritiker") versuchen wir, noch einen Schritt weiterzugehen: nämlich jene erkenntnistheoretische Position zu erfassen, die den in ihrem Aùfsatz weitgehend unkenntlich gelassenen Fluchtpunkt der Kritiker ausmacht, deren Spuren aber gesichert werden können. Damit hoffen wir zugleich, Hinweise für die Lösung des Rätsels zu erhalten, welchen - und hier dann schließlich unmittelbar politischen - Ursachen und Motiven sich das von uns untersuchte Resultat von Fälschung oder selektiver Wahrnehmung verdankt (4).

Da die Kritiker die Substanz der materialistischen Abbildtheorie, nämlich den Materialismus, nicht begriffen haben und ihr Nichtbegreifen mit Fälschungen zu legitimieren suchen, handelt es sich also für uns um die Rekonstruktion dieser materialistischen Substanz. Zu einzelnen Fragen der Abbildtheorie gibt es - allerdings auf Grundlage dieser identischen Substanz -, durchaus unterschiedliche Antworten innerhalb der marxistischen Diskussion und offene Probleme, auf die wir hier, da jenseits der Auseinandersetzung mit den Kritikern liegend, nicht eingehen. Vielleicht ist in diesem Zusammenhang der Hinweis angebracht, daß auch der "Sowjetmarxismus" nicht so "monolithisch" ist, wie man seit Adenauers Zeiten hierzulande glaubt. Die Kritiker führen (152, Anm. 1) nur drei in Westdeutschland erschienene Abhandlungen zur materialistischen Abbildtheorie an. Es gibt einige mehr.

Um späteren Ausführungen (vgl. Teil I, 2) vorzugreifen: es ist auch gleichgültig, ob es sich um bewußte Fälschung oder (unbewußt) selektive Wahrnehmungen handelt - falsches Bewußtsein äußert sich darin jedenfalls, und zwar eben objektiv falsches als Form jener verzerrten Widerspiegelung, die die Abbildtheorie, den Kritikern zufolge, nicht kennt. -

$+++$

Im zweiten Teil ihres Aufsatzes setzen sich die Kritiker mit Autoren der Zeitschrift „Das Argument" (Haug, Tomberg) auseinander. Darauf werden wir nicht eingehen. Zwar wird die Kritik an ihnen nicht minder fahrlässig geführt; für die Analyse der Motivstruktur, die zu der ganzen Invektive führte, wäre sie eine Fundgrube. Wir verzichten dennoch auf den exakten Aufweis dieses Tatbestandes und empfehlen dem Leser, einfach die inkriminierten Aufsätze von Haug und Tomberg im Original nachzulesen; das ist zur Beurteilung der „Kritik" völlig hinreichend. In der Substanz aber ist der zweite Teil des PROKLA* Aufsatzes ohnehin nicht zu halten, wenn der erste als haltlos erwiesen ist. 


\section{Die Kritik der Kritiker}

Wir rufen in Erinnerung, was die Kritiker der Abbildtheorie anlasten:

„Die Abbild theorie als die gegenwärtig in den sozialistischen Staaten herrschende Erkenntnistheorie destruiert nicht, sondern festigt die bürgerliche Wissenschaftsauffassung. Sie befestigt den Schein, Wissenschaft sei eine Veranstaltung des menschlichen Geistes, unabhängig von der Form der Vergesellschaftung, die objektive Form der Erfahrung habe nicht ökonomische, sondern natürliche Ursachen, Erkenntnistheorie habe es mit einer überhistorischen MenschNatur-Relation zu tun und sei folglich als Sonderdisziplin neben und unabhängig von der politischen Okonomie zu berreiben" (152). (5)

Unabhängig von den Einzelheiten dieser Behauptungen, auf die wir zu sprechen kommen müssen, wird hier der schärfste Vorwurf ausgesprochen, den man einem im Selbstverständnis marxistischen Theorem machen kann, nämlich Moment bürgerlicher Ideologie zu sein. Wer einen derart enormen Vorwurf erhebt, muß gewärtigen, daß man ihm auf die Finger sieht.

Wenn wir uns und den Leser deshalb der Mühe unterziehen, die Argumentierund Zitierweise der Kritiker, die sich so schlagend und schlicht gibt, auszuleuchten, so gewiß nicht, um formale Mängel zu rügen. Dieses oder jenes Zitat als überinterpretiert oder zu Unrecht herangezogen aufzuweisen, würde den Aufwand nicht lohnen. Es handelt sich aber nicht um dieses oder jenes, sondern um alle, und daher für uns nicht um Schulmeisterei, sondern um die Aufdeckung durchgehender Fälschung. Wir kommen nicht umhin, dieses harte Wort zu gebrauchen: denn was im Detail vielleicht noch als Unterstellung, Auslassung, Verwischung der Zusammenhänge, Verschiebung der Bezugsebenen usw. in und von Zitaten hinzunehmen wäre, wird in der Summierung, die keinen einzigen der angeblichen Belege ausläßst, zur - übrigens, wie zu zeigen sein wird, einer eigenen Logik folgenden - Fälschung.

Dieses Zitat ist im Original als zusammenfassende These hervorgehoben. 


\section{Die Quelle}

Die Kritiker lassen „,bewußt außer Acht“, daß die „Abbildtheorie auf Lenin (u. in Teilen schon auf Engels)" zurückgeht. Sie konzentrieren sich auf das Philosophische Wörterbuch (6), das die Abbildtheorie ,in ihrer heutigen Form" vorstelle und wegen seiner beängstigenden Verbreitung in der linken akademischen Intelligenz besonders schädlich sei (153, Anm. 5) (7). Bereits diese Begründung der Beschränkung auf das Philosophische Wörterbuch (Phil.W.B.) enthält - abgesehen von der offenkundigen Überschätzung der Relevanz studentischer Kopfinhalte für den Klassenkampf - zwei Insinuationen. Erstens, daß die Abbildtheorie sich zwar zu Recht auf Lenin und z.T. auch auf Engels berufen könne, nicht aber auf Marx. (8) Wir erkennen darin ein altes, unter „Marxologen“ und „Marxianern“ aller Spielarten liebevoll gehegtes Muster der Klassikerbehandlung: das Auseinanderdividieren von Marx und Engels, vollends Lenin (9). Zweitens wird mit der Formulierung „,heutige Form“ nahegelegt, diese unterscheide sich von fruiheren, so daß also, trotz auch wieder betonter Identitäten in den Ansichten von Lenin und dem Phil.W.B. (vgl. 153), die heutige Form nochmals als Verfallserscheinung Leninscher und Engelsscher Auffassungen dasteht. So wird ein unmittelbarer Angriff auf Engels und Lenin vermieden, was sich angesichts dessen empfehlen mag, daß beide auch in der „nichtrevisionistischen“ Linken noch weithin sakrosankt sind.

6 Philosophisches Wörterbuch, hrsg. von Georg Klaus und Manfred Buhr, 2 Bände, 8. Aufl., Leipzig 1971

7 Als weitere Begründung wird noch der repräsentative Charkater des Phil. W.B. angeführt: ,,in ihm sind die namhaftesten Theoretiker der DDR vertreten“. Folgt ein Seitenhieb auf den nicht-pluralistischen Charakter der DDR-Gesellschaft: während sich im Westen die Theorie-Selektion ,über den Markt" herstelle, geschehe in einem sozialistischen Staat wie der DDR die „,Produktion und Verbreitung von Theorien ... planvoll“. Wir schließen aus diesem Hinweis, daß, da die planvolle Produktion und Verbreitung mindestens im Falle der Abbildtheorie Falschmeldungen dient, also schädlich ist, die Markt-Sele ktion immer noch vorzuziehen sei. Die freie Marktwirtschaft enthält eben größere Chancen der Wahrheitsverbreitung. Freilich hätten die Kritiker ihre Auffassungen in der ,totalitären“ DDR kaum publizieren können. Die Marktwirtschaft der BRD zeitigt andererseits oft kaum überschaubare Ergebnisse. So könnte es sein, daß wir mit unserem Nachweis der Haltlosigkeit der Auffassungen der Kritiker deren Marktwert steigern.

8 Folgerichtig werden dann auch sämtliche Marx-Zitate, die sich im Phil. W.B. im unmittelbaren Kontext von Stellen finden, die die Kritiker anführen, schamvoll unterschlagen. Inwiefern selbstverständlich auch Marx Widerspiegelungstheoretiker ist, dazu vgl. unten Teil II, 2, sowie Anm. 33.

9 Mit Engels, von Lenin zu schweigen, ist ein kruder Positivismus in das kritische Denken des Marxismus eingedrungen. Genau besehen, ist er, als ,, heimlicher Positivismus“ bereits beim späteren Marx zu diagnostizieren (vgl. z. B. A. Wellmer, Kritische Gesellschaftstheorie und Marxismus, Frankfurt/M. 1969). Wirklich an die Wurzeln gehende, also radikale Interpreten nehmen deshalb an Marx selbst nochmals eine Zellteilung vor und unterscheiden den frühen kritischen (den Marx der emphatischen Entfremdungskategorie) vom späten positivistischen (dem Anatomen der bürgerlichen Gesellschaft). Die amüsante Geschichte des Klassikerdividierens, in der die progressiven protestantischen Theologen eine ebenso große Rolle spielen wie die kritischen Theoretiker der Frankfurter Schule, wartet noch auf ihren Historiker. 
Andere Vorteile der Auswahl des Phil.W.B. als Demonstratonsobjekt schein uns hinsichtlich der Beweisabsicht der Kritiker einleuchtender. Zum einen ist ein Wörterbuch kein zusammenhängender Text, der seine Positionen argumentativ entfaltet, sondern ein alphabetisch geordnetes Konglomerat knappster Ergebnisdarstellungen, immer auf definitorische Pointen abgestellt (10). Man kann also, das garantiert das literarische Genre Wörterbuch, mit einem geringen argumentativen Differenzierungsgrad rechnen, was sich, richtig beleuchtet, als Dogmatismus vermitteln läßt. Zum anderen referiert ein Wörterbuch nicht nur Auffassungen, die seine Autoren - selbst wenn sie untereinander eines Sinnes wären - teilen, sondern auch abweichende und entgegengesetzte; zudem werden allgemeine Definitionen an den Anfang jedes Artikels gestellt, die den Minimalkonsens aller Auffassungen eines Zusammenhangs treffen sollen und ebenfalls mit den Positionen der Verfasser nicht identisch sein müssen; daraus ergeben sich reichhaltige Zitationsmöglichkeiten gegen deren wirkliche Meinung.

Wir wollen nun weder vertreten, daß ein Wörterbuch nicht kritisierbar sei; nur muß man seine Eigenart dabei berücksichtigen (11). Noch wollen wir unterstellen, daß die genannten Motive für die Kritiker bei der Quellenwahl maßgebend waren. Sie haben jedoch die sich daraus ergebenden Möglichkeiten reichlich genutzt: Zitiert wird aus mehreren Artikeln des Wörterbuchs, ohne deren Auswahl zu begründen (12); die Auffassungen der verschiedenen Verfasser werden dabei als selbstverständlich identisch angesehen; zitiert wird aus verschiedenen Passagen der einzelnen Artikel so, als hätten sie für die Auffassungen ihrer Autoren alle die gleiche Relevanz. Das Wörterbuch wird, kurz gesagt, traktiert, als wäre es eine zusammenhängende Darstellung der Probleme der Abbild- oder Widerspiegelungstheorie.

10 Ein Standardwerk wie das von Todor Pawlow, Die Widerspiegelungstheorie, deutsch: Berlin (DDR) 1973, 757 SS., hätte freilich eine so flotte Vernichtung gar nicht erst erwägbar gemacht. Denn darin ist der Zusammenhang hergestellt, der im Phil. W.B. auf viele Beiträge zersplittert ist, und es werden zudem, was in einem Wörterbuch nicht geschehen kann, die wirklichen Probleme erörtert, denen sich eine materialistische Erkenntnistheorie konfrontiert sieht. Von den Kritikern wird dieses Werk übrigens in der entsprechenden Anmerkung nicht erwähnt (152, Anm. 1).

11 Durchaus wäre am Phil. W.B. manches zu kritisieren. Mängel in der terminologischen Exaktheit, zu grobe Darstellungen, auch wirkliche Fehler, lassen sich aufweisen, und ihre Kritik ist auch bei Berücksichtigung seiner Eigenart als Wörterbuch berechtigt. Diese Ebene betreten die Kritiker aber nicht, und soweit ihre Kritik reicht, hat das Wörterbuch stets noch da Recht, wo es unter anderen Gesichtspunkten Fehler enthält.

12 Die Zitate stammen, meist ohne daß dies kenntlich gemacht würde, aus folgenden Artikeln: „Abstraktion", „Bewußtsein", ,Denken", „Erkennbarkeit der Welt", ,Grundfrage der Philosophie“, „Intersubjektivität", „Sinne“, ,Praxis". Oft wird nur ein einziger Satz zitiert (der definitorische Anfang). Nicht minder einschlägig wären einige Artikel, die die Kritiker nicht heranziehen: merkwürdigerweise finden sich offenbar weder in dem Artikel „Abbildtheorie“, noch in dem Artikel „Widerspiegelung" Belegstellen im Interesse der Kritiker; auch in anderen nicht ganz nebensächlichen Artikeln findet sich wohl nichts Zitierenswertes, z. B., um nur beliebig einige zu nennen, in den Artikeln „Gesellschaftliches Bewußtsein", „Ideologie", „Ideologienlehre", ,Materialismus", "Objektivität". 
Aus den ohnehin argumentativ notwendig verkürzten Texten werden nunmehr Zitate immer so ausgewählt, daß der gesellschaftliche Zusammenhang von Bewußtseinsbildung und Erkenntnisprozeß, der im Wörterbuch sehr stark betont wird, ausgeblendet bleibt. Für Leser, die so pingelig sind, die Zitate zu überprüfen, wird vorbeugend eine pauschale Absicherung geliefert: „Die Gesellschaft als Bestimmungsgröße taucht in der Abbildtheorie immer nur programmatisch auf und dabei eigentümlich verkürzt“" (156). Als könnte in einem Wörterbuch die „Gesellschaft als Bestimmungsgröße" je historisch-spezifisch entwickelt werden! Soweit die Eigenart eines Wörterbuchs es aber zuläßt, erscheint diese Bestimmungsgröße als zentrale Instanz, und zwar nicht ,programmatisch“, also als etwas, das man nicht ausführt oder ausführen kann, sondern als theoretische Position. Die ,eigentümliche Verkürzung“ verdankt sich einzig der Zitierkunst der Kritiker. Hinzu kommt, daß die so ausgewählten Zitate nicht einmal tauglich sind, das zu belegen, was die Kritiker interpretatorisch glauben, aus ihnen herausholen zu können (13). Wir zeigen das nun im einzelnen.

\section{Die Objektivität der Widerspiegelung und der Gegenstand der Widerspiege- lungstheorie}

An ein berühmtes Lenin-Zitat (,,kopiert, fotografiert, abgebildet") und ein in der Substanz ähnliches aus dem Phil.W.B. (153) als Belege schließen die Kritiker die Behauptung an, die Abbildtheorie projiziere „die objektive Form von Erkenntnis als scheinbare Naturform auf die ganze Geschichte zurück" (154). Was immer dieser Satz bedeuten mag: von den angeführten Zitaten würde er jedenfalls nicht gedeckt. Aber was bedeutet er?

\section{1, Objektiv" bei den Kritikern}

Wir müssen uns vor allem vergewissern, was die Kritiker unter ,objektiv“ verstehen, und ob es dasselbe ist, was die Abbildtheorie damit meint. „Wahrheit", so schleudern sie der Abbildtheorie im Zusammenhang ihrer Zerstörung des abbildtheoretischen Begriffs der „relativen Wahrheit" entgegen, ,gibt es nicht in Steigerungs-

13 Die Kritiker ahnen dies wohl, denn sie haben sich vorsorglich eine Rückzugslinie geschaffen: im Grunde ,sind die Fehler der Abbildtheorie und ihr genuin undialektischer Charakter ... bereits so überzeugend nachgewiesen worden, daß wir diesen Nachweis nicht in extenso wiederholen müssen" (152). Folgt eine Fußnote mit Literaturhinweisen, also eine Absicherung mit Autoritäten: dem frühen Lukàcs, Korsch, Pannekoek und Negt, auf deren ,Widerlegungen" wir hier nicht eingehen können. Doch findet sich z. B. in der angegebenen Schrift von Negt wiederum kein Versuch einer Widerlegung, sondern nur die Absicht, die Abbildtheorie als stalinistische Legitimationsideologie nachzuweisen, im übrigen der Hinweis, die Abbildtheorie sei eine "mittlerweile als falsch erkannte Theorie", also jene Behauptung, die die Kritiker zitieren (167). 
formen“ (162). Und sie erläutern in der Fußnote: „Es gibt nicht objektiา objektiver, am objektivsten" (162, Anm. 20). Auf die Frage der relativen Wahrheit kommen wir später (14). Zunächst ist an diesen Wendungen wichtig, daß in ihnen „objektiv" als gleichbedeutend mit ,wahr" im Sinne von „,richtig" aufgefaßt wird. Das bestätigt sich in der ständigen Bindung des Wörtchens „objektiv“" an ,wissenschaftlich“ und an „Erkenntnis“ (z.B. „Sachverhalt objektiver und wissenschaftlicher Erkenntnis“, 162). Wahr, wissenschaftlich, objektiv: das sind den Kritikern, so ergibt sich aus ihrem Sprachgebrauch ganz zweifelsfrei, Worte, die das gleiche meinen: nämlich richtige Aussagen über Sachverhalte, und diese wieder sind dasselbe wie Erkenntnis.

Die Bedeutung des oben zitierten Satzes der Kritiker wird damit klarer. Die „objektive Form von Erkenntnis" ist die wahre, die wissenschaftliche, die richtige Form von Erkenntnis, und eigentlich hätten die Kritiker bloß sagen dürfen: „die Form von Erkenntnis", denn wahr, wissenschaftlich, richtig, objektiv sind ja in Verbindung mit „Erkenntnis“ pleonastische Ausdrücke, da es unwahre, unrichtige, unwissenschaftliche, nicht-objektive Erkenntnis in diesem Verstande des Begriffs nicht geben kann. Die Abbildtheorie soll also, das meinen die Kritiker, den an den modernen Naturwissenschaften orientierten Begriff von Erkenntnis auf alle Geschichte zurückprojizieren, und zwar als ,Naturform" (15), will sagen: daß die Menschen seit Anbeginn durch die natürliche Beschaffenheit ihrer Sinnesorgane gar keine anderen sinnlichen, mentalen, intellektuellen Reaktionen auf ihre natürliche und soziale Lebenswelt zustande bringen konnten, als eben richtige, wahre, wissen-

schaftliche und in diesem Sinne objektive Widerspiegelungen, also schlechthin Erkenntnis. Dies sei abbildtheoretisch ,die Normalreaktion des homo sapiens auf die Natur" (161). Bis hin zu der entlarvend-ironischen Frage an die Abbildtheorie, ob Ptolemäus doch recht hatte (162, Anm. 20), lebt die ganze Polemik der Kritiker von diesem simplen Verständnis des Begriffs „objektiv“ und den daraus sich ergebenden Interpretationen. Und wer würde denn den Kritikern, hätten sie damit recht, bestreiten wollen, daß solche Abbildtheorie absurd sei? Erstaunlich wäre dann nur, wie das nicht schon früher und jedermann auffallen konnte.

\subsection{Verscbiebungen}

Wir werden gleich darauf kommen, was die Abbildtheorie wirklich unter „,objektiv“ versteht, müssen aber noch einen Moment bei den Interpretationen der Kritiker verweilen. Denn man könnte ja sagen: nun, hier handelt es sich offenbar um

s. u. I, 4

Immer wieder tauchen bei den Kritikern die Begriffe „Erkenntnisform“", „Naturform“, „,Objektform" auf. Die Bedeutung dieser Begriffe ist nicht so selbstverständlich und landläufig, wie die Kritiker voraussetzen, indem sie sie nicht näher erläutern. Wir meinen jedoch, daß hier ein recht genaues, aber esoterisches Begriffsverständnis vorliegt, dessen Provenienz wir im Teil II, 3 zu erfassen suchen. 
Differenzen um das Begriffsverständnis von „objektiv“, darüber wird man wohl noch diskutieren dürfen, ohne gleich unter Fälschungsverdacht zu geraten. Das wäre gewiß richtig, aber darum handelt es sich nicht. Die Kritiker sehen nämlich gar keine Differenz zwischen ihrem eigenen Begriff von „objektiv“" und den Verwendungen des Wortes in den Zusammenhängen der Widerspiegelungstheorie. Unbefangen und wie selbstverständlich gehen sie davon aus, kein Mensch, einschließlich derer, die der Widerspiegelungstheorie anhängen, habe je unter „objektiv" etwas anderes verstanden als sie selber. Und wenn sie nun - übrigens nur aufgrund allzuflüchtiger Lektüre; dazu später - im Phil.W.B. für diesen, ihnen einzig denkbaren Sinn des Wortes „objektiv“ kein Beleg finden, sondern nur Verwendungen antreffen, die einem vorm Hintergrund jener zweifelsfreien Eindeutigkeit des Wortsinnes bloß absonderlich, verschroben und ungereimt erscheinen können -: dann kommt man als scharfsinniger Kritiker nicht etwa auf den Gedanken, etwas nicht verstanden zu haben. Schon gar nicht fühlt man sich in der Klemme von Beweisnot. Umgekehrt: wenn die Belege fehlen, dann ist eben ihr Feblen der Beleg.

„Am deutlichsten äußert sich diese Rückprojektion (der objektiven Erkenntnis als Naturform des Denkens auf die ganze menschliche Geschichte; d. Verf.) darin, daß in der Abbildtheorie das Attribut ,objektiv' nicht ein erläuterndes Beiwort von Erkenntnis ist, sondern immer der Realität als Eigenschaft zugeschlagen wird. So hieß es in dem Zitat nicht, alle Erkenntnis sei eine ,objektive Widerspiegelung der Realität', sondern, in charakteristischer Verschiebung, sie sei eine ,Widerspiegelung der objektiven Realität" " (155). (16)

So wird noch das Gegenteil dessen, was man beweisen will, zum schlagendsten Indiz der Anklage, so, wie man ja auch, Karl Kraus zufolge, seinerzeit im KK-Wien die Mörder der Lüge überführt wußte, wenn man ihnen den Mord nicht beweisen konnte. Ausgehend davon, daß die Widerspiegelungstheoretiker selbstverständlich unter „objektiv“" nichts anderes als jedermann und sie selber verstehen, haben die Kritiker derart „bewiesen“, daß die Widerspiegelungstheorie diesen Begriff nicht erträgt, nicht zuläßt, daher seinen Sinn ,verschieben“ muß, um jenen uferlosen Relativismus zu kaschieren, den die Kritiker ja als theoretisches Substrat der Widerspiegelungstheorie entlarven wollen.

Was also wird von wem ,charakteristisch“ verschoben und mit welchem Ergebnis? In dem eben angeführten Diktum der Kritiker wird auf ein von ihnen herangezogenes Zitat aus dem Phil.W.B. Bezug genommen. Es stammt, was die Kritiker charakteristischerweise nicht kenntlich machen (17), aus dem Artikel „Erkenntnis“ und bezieht sich mithin nicht auf Bewußtseinsinhalte schlechthin, sondern auf die besonderen Formen wissenschaftlicher Erkenntnis. Verschoben wird von den Kritikern also zunächst einmal die Bezugsebene: während in dem

16 Hervorhebung d. Verf.. Der darauf folgende konkludierende Satz heißt dann lakonisch: ,Die Objektform wird als ewig seiende Naturqualität unterstellt" ${ }^{*}$. Wir deuten mal so: Die Wahrheitsform des Denkens wird als ewig seiende Qualität der menschlichen Natur unterstellt.

17 Vgl. zu diesem Verfahren oben Anmerkung 12 
Artikel von einer spezifiscben Form der Bewußtseinstätigkeit, nämlich der „Erkenntnis“ und ihrem Resultat als Wissen die Rede ist, von der gesagt wird, sie sei „,ihrem allgemeinen Wesen nach eine annähernd getreue Abbildung oder ideelle Wiederspiegelung der objektiven Realität im Bewußtsein der Menschen“ (18), insinuieren die Kritiker, laut Abbildtheorie sei alle Bewußtseinstätigkeit der Menschen in aller Geschichte Erkenntnis, mithin ,annähernd getreue Abbildung“ (19) der objektiven Realität. Dies ist die erste charakteristische Verschiebung der Kritiker (20), denn natürlich behauptet kein Abbildtheoretiker die Absurdität, alles Bewußtsein in aller menschlichen Geschichte sei Erkenntnis im modernen Sinne, mithin richtige, adäquate Widerspiegelung objektiver Realität.

Die zweite ist jene, die die Kritiker der Abbildtheorie als deren eigene unterstellen: die ,charakteristische Verschiebung“ der „Objektform“ (155) der Widerspiegelung auf die Realität. In Wahrheit also - so die Kritiker - behauptet die Abbildtheorie die „Objektform" der Widerspiegelung, verschleiert dies aber - wohl um nicht allzu leicht der Absurdität überführbar zu sein, was ja dann auch die Mühen der Kritiker erübrigte -, indem sie diese als „Objektform“ der Realität ausgibt. Wir sagten bereits, daß sich diese, ohne weitere argumentative Umstände wie selbstverständlich unterstellte Verschleierungsabsicht nur dann überhaupt formulieren und verstehbar machen läßt, wenn der Begriff ,objektiv“ im Sinne der Kritiker-Bedeutung verstanden wird. „Objektform" wäre also mit „Wahrheitsform“ zu übersetzen und die Abbildtheorie hätte demnach die Wahrheit der Widerspiegelung - der Realität als Attribut „zugeschlagen“. Soweit die Verschiebungen.

\section{3 „Objektiv“ in der Widerspiegelungstheorie}

Was heißt nun jedoch "objektiv“ in der materialistischen Erkenntnistheorie? Zunächst und vor allem anderen wird mit diesem Begriff ausgesprochen, daß das natürliche und soziale „Sein“ unabhängig vom Bewußtsein, das die Menschen davon haben, existent ist, daß also Realität etwas ist, was nicht erst durch menschliche Hirntätigkeit bergestellt wird. „Objektiv“" ist folglich in diesem Sinne ein Begriff, der idealistische Auffassungen von den Erkenntnisgegenständen negiert. Objektiv ist die Existenz der Realität, weil sie nicht das Produkt mentaler Operationen menschlicher Subjekte ist. Die Begriffsbedeutung von „objektiv“" hat hier demnach nichts zu tun mit Wahrheit, Richtigkeit, Intersubjektivität und Wissenschaft im modernen

Phil. W. B. I, 315

,annähernd“" bedeutet soviel wie „relativ“. Wir befassen uns damit später (I, 4).

Die Kritiker reproduzieren diese Verschiebung in immer neuen Varianten: die Abbildtheorie behaupte ,eine wesentliche Identität aller Erkenntnisformen" (154); sie setze „die objektive Erkenntnisform und die Existenz eines allgemeinen Bewußtseins (! - d. Verf.) ,der" Menschen naturhaft" (157) voraus; sie unterstelle „Erkenntnisse in immer gleicher objektiver Form" und sie lasse den Schein entstehen, „die objektive Form von Erkenntnis sei die Naturform von Erkenntnis" (161). 
Sinne. Es ist ganz gleichgültig, ob man die Wirklichkeit als wahr, falsch, gut, schön, richtig oder irre ansieht: objektiv ist sie, weil von derartigen Anschauungsweisen und Urteilen unabhängig existent. Die Kritiker dagegen meinen, das Wort „objektiv" werde von den Abbildtheoretikern „der Realität als Eigenschaft zugescblagen " (!). Wer einer solchen Formulierung fähig ist, der verrät nicht nur, daß er der primären Bedeutung des Begriffs ,objektiv" in der Widerspiegelungstheorie nicht innegeworden, sondern zugleich auch, daß er nicht weiß, was Materialismus ist.

Nun hätten die Kritiker freilich, bei näherem Hinsehen, durchaus auch Belegstellen finden können für jenen anderen Bedeutungsgehalt von ,objektiv“, den die Widerspiegelungstheorie angeblich stets verschiebt. An einschlägiger Stelle des Phil.W.B. wird dieser Sprachgebrauch legimitiert. Wir sprechen, heißt es da

„im Zusammenhang mit der mensehlichen Erkenntnis . . von ihrem objektiven Inhalt und Charakter, von ihrer Objektivität. Die Objektivität der Erkenntnis besteht darin, daß das Subjekt die objektive Realität adäquat widerspiegelt, daß die Erkenntnis mit dem Objekt übereinstimmt" (21).

Hier wird „objektiv“" zweifellos als „erläuterndes Beiwort von Erkenntnis“ benutzt, ein ganz uncharakteristischer Gebrauch, wenn man den Exegesen der Kritiker Glauben schenken will. Tatsächlich handelt es sich aber um eine auch in den Texten der Widerspiegelungstheorie und daher auch im Phil.W.B. geläufige Bedeutung. Die Kritiker hätten sich also den Umweg über die „charakteristische Verschiebung“" gar nicht machen müssen; die Abbildtheoretiker sagen es selber ganz ungeniert: Erkenntnis sei objektiv.

Halten wir fest: es gibt zwei Bedeutungen von „objektiv“ in der Sprache der Widerspiegelungstheorie, die nebeneinander benutzt werden, jeweils aus dem Kontext zu bestimmen sind und verschiedenen Sprachstufen angehören. Man kann das an der wissenschaftstheoretischen Unterscheidung zwischen Objektsprache und Metasprache verdeutlichen: soweit „objektiv“ sich auf die Realität selbst bezieht, indem deren subjektunabhängige Existenz gemeint ist, ist es ein objektsprachlicher Ausdruck; soweit „objektiv“ sich auf Aussagen über die Realität bezieht, indem deren Wahrheit oder Erkenntnischarakter behauptet wird, ist es ein metasprachlicher Ausdruck. Beide Begriffsbedeutungen haben ihren Gegensatz in „subjektiv“: die objektsprachliche, indem sie jede Form der idealistischen Konstitution der Erkenntnisgegenstände verwirft und diese als subjektunabhängig bestimmt; die metasprachliche, indem sie die Differenz etwa zu den "Wahrheiten" individueller Gefühle ausdrückt.

Obwohl diese Unterscheidung zwischen objektsprachlicher und metasprachlicher Bedeutung des Begriffs dem Verständnis der Kritiker bei der Rezeption der Widerspiegelungstheorie vielleicht geholfen hätte, leistet sie an sich noch nicht viel; ihrer Herkunft aus der bürgerlichen Wissenschaftstheorie gemäß bleibt sie deskriptiv. Die Dialektik der materialistischen Begriffs „objektiv“", wie er die Widerspiege- 
lungstheorie bestimmt, wird erst jenseits dieser Unterscheidung verstehbar. Die Begriffsbedeutung verwandelt sich je nach Gesichtspunkt und Bezugsebene. So steht „objektiv“ zwar stets im Gegensatz zu "subjektiv“. Deshalb ist aber „Objektivität" durchaus nicht notwendig gleich "Intersubjektivität", wie die Kritiker ja wohl meinen, wenn sie von ,Allgemeingültigkeit oder ,Intersubjektivität von Erkenntnis ..." (158) sprechen: das Allgemeinguiltige ist nicht notwendig identisch mit dem Allgemeingeltenden, der Fall ist eher selten. Objektiv ist nicht stets das, was wahr ist, aber Erkenntnis ist stets objektiv.

Derlei mag paradox klingen. Prüft man aber die Relation, von der die Begriffsbedeutung jeweils bestimmt ist, so verliert sich das. In der Relation zwischen Subjekt und Objekt der Erkenntnis heißt „objektiv" zum einen die subjektunabhängige Existenz des zu erkennenden Objekts, zum anderen die Adäquatheit der Widerspiegelung dieses Objektiven durch das erkennende Subjekt: die Widerspiegelung ist objektiv, also Erkenntnis, wenn sie die objektive Realität adäquat abbildet, sie ist dann wabr. Andererseits kann die Widerspiegelung der objektiven Realität auch falsch sein, verzerrt, und dennoch objektiv: sie ist dann nicht objektiv, weil wahr, sondern objektiv, weil subjektunabhängig existent: hier erhält der Begriff seine Bedeutung innerhalb der Relation zwischen individuellem und gesellschaftlichem Bewußtsein. Handelt es sich um die „Wahrheit" eines individuellen Gefühls, so ist dieses zwar gewiß real existent, aber subjektiv, da es an ein individuelles Bewußtsein gebunden ist; es handelt sich um eine subjektive Realität. Handelt es sich aber um kollektive Meinungen und allgemein herrschende Vorstellungen, deren Existenz mithin nicht von einen individuellen Bewußtsein abhängig ist, so sind diese objektiv; es handelt sich dann um ideelle Momente der objektiven Realität selbst. Und wenn nun ein solches ideelles Moment der objektiven Realität zugleich auch Wahrheit ist, weil adäquate Widerspiegelung der objektiven Realität, also Erkenntnis, der Objektivität zukommt, dann ist es im doppelten Sinne objektiv, nämlich einerseits als subjektunabhängiger, gesellschaftlich allgemeiner Bewußtseinsinhalt, andererseits als Erkenntnis; es ist zugleich objektiv real und objektiv wahr. Der Begriff ,objektiv“"kann also sowohl die Bedeutung von ,objektiv real“, als auch die von ,objektiv wahr" annehmen, wobei beide Bedeutungen sich nicht ausschließen, sondern auch zugleich gemeint sein können.

Der Begriff einer adäquaten Widerspiegelung objektiver Realität wäre sinnlos und überflüssig, behauptete die Abbildtheorie tatsächlich, wessen die Kritiker sie überführt zu haben meinen: alles Bewußtsein sei Erkenntnis, der Objektivität zukomme. Selbst ein Abbildtheoretiker, wie er den Kritikern vorschweben mag, könnte kaum übersehen, daß in Geschichte und Gegenwart - um im Bilde zu bleiben: - verzerrte Widerspiegelung, falsches Bewußtsein, nicht-adäquate Abbildung der objektiven Realität die Hauptmasse der Inhalte menschlichen Bewußtseins und der ihnen entsprechenden Formen ausmacht. Freilich sind, ohne daß sich dies mit der Frage ihrer Wabrbeit im Sinne adäquater Widerspiegelung berührte, alle Formen von Bewußtsein, die die Menschheit je hervorgebracht und gezeitigt hat, und noch ihre scheinbar abwegigsten Ein-Bildungen, soweit sie überindividuelle, also gesellschaftliche Bedeutung erlangt haben, wie gesagt in dem Sinne wiederum 
objektiv, als sie selbst - ideelles - Moment der objektiven Realität sind (22). Dies sind sie natürlich alle gleichermaßen: nicht einmal unter Aufbietung von Mißverständnissen läßt sich diese Bedeutung von ,objektiv" als steigerungsfähig verstehen. Etwas anderes ist es bei der Bedeutung von ,objektiv" im Sinne von ,wahr": hier sind die Kritiker dem Irrtum zum Opfer gefallen, der abbildtheoretische Begriff der ,relativen Wahrheit" meine: etwas weniger objektive Wahrheiten; aber ,relativ" steht hier nicht im Gegensatz zu „objektiv" (dessen Gegensatz, wie bemerkt, „subjektiv" ist), sondern im Gegensatz zu ,,absolut" (doch dazu später).

Der Begriff ,objektiv" in der materialistischen Widerspiegelungstheorie ist, so zeigt sich, in jeder Bedeutung, die er in ihrem Zusammenhang annehmen kann, gebunden an den primären Sinn der Bezeichnung für die subjektunabhängige Existenz der Realität. Auch da, wo „objektiv" soviel wie „wahr" heißt, besteht daher nur eine äußerliche, formale Übereinstimmung mit der Bedeutung des Wortes im Sprachgebrauch der Kritiker, der ja übrigens der landläufige ist. Dieser Sprachgebrauch ist zwar auch in dem der Widerspiegelungstheorie enthalten, aber mehr noch: er ist darin ,aufgehoben“, indem er der begrifflichen Fassung der realen Dialektik von materiellen und ideellen Momenten der subjektunabhängigen Wirklichkeit dient. Wird abbildtheoretisch von Objektivität der Erkenntnis gesprochen, so ist das nicbt pleonastisch, denn es wird mehr gesagt, als daß Erkenntnis Wahrheit sei. Zugleich und in einem wird nämlich stets auch gesagt, warum sie Wahrheit ist und nur sein kann: nämlich weil sie die vom erkennenden Subjekt unabhängig existierende Wirklichkeit adäquat widerspiegelt (eine andere, im gegenwärtigen Zusammenhang nebensächliche, sonst aber sehr wesentliche Frage ist, warum und unter welchen Bedingungen es $\mathrm{zu}$ adäquater Widerspiegelung, also Erkenntnis, kommen kann). Dies ist der springende Punkt der materialistischen Widerspiegelungstheorie, und wir müssen kaum nochmals erwähnen, daß es eben jener Punkt ist, der sich bei den Kritikern bloß als blinder Fleck abbildet.

Bindet man den Begriff der Erkenntnis nicht borniert an die Auffassung moderner Wissenschaftlichkeit, so läßt sich wohl vertreten, daß auch scheinbar ganz abwegige Bewußtseinsinhalte, z. B. die von den Kritikern angeführten "magischen Vorstellungen früher Epochen" (161, Anm. 19), nicht nur objektiv real sind - und auf der jeweiligen historischen Stufe der Entwicklung der Produktivkräfte objektiv notwendig -, sondern auch Erkenntnischarakter haben können. Es ist jeweils zu untersuchen, welchen Wahrheitsgehalt eine Vorstellung hat, wo ihre Grenzen liegen, $d$. h. wie relativ sie ist. Natürlich können die Grenzen außerordentlich eng sein (wobei man mit "Grenzen" nicht eine mechanisch-räumliche Vorstellung verbinden sollte). Ein Beispiel: Ein archaisches Hirtenvolk erklärt sich einen feuerspeienden Vulkan mit dem Zorn eines Gottes, der darin haust. Dies ist allerdings keine wissenschaftliche Wahrheit: als Aussage über einen Kausalzusammenhang ist sie falsch. Aber es steckt darin u.a. die Erkenntnis, daß ein Kausalzusammenhang vorliegt; dies ist die relative Wahrheit einer falschen Aussage uber Wirklichkeit. Ihre Relevanz liegt, wie bei aller Erkenntnis, in der Praxis: die falsche Annahme eines zürnenden Gottes führt, abgesehen von allerlei religiösem Unsinn, zur Beobachtung des Vulkans, um das Zürnen voraussagen zu können und seinen Folgen zu entgehen. 


\subsection{Erkenntnis und Erkenntnisgescbicbte}

Die Kritiker können die Dialektik des Begriffs „objektiv“ in der materialistischen Widerspiegelungstheorie nicht fassen. Es ist ihnen daher auch verwehrt einzusehen, daß Bewußtsein zugleich - gemessen am Kriterium der Adäquatheit von Widerspiegelung - objektiv falscb, und dennoch, hinsichtlich seiner Gesellschaftlichkeit, objektiv, nämlich objektiv real, sein kann. Daraus resultiert das Unvermögen, die Problematik des Ideologischen von der der Erkenntnis zu unterscheiden. Freilich gibt es in der marxistischen Theorie Bedeutungsvarianten dessen, was Ideologie heißt. Die Dialektik des Begriffs ,objektiv“, die wir zu zeigen versucht haben, reproduziert sich hier. Allen Bedeutungen von Ideologie ist allerdings gemeinsam die kritische Distanz zu idealistischen Auffassungen ideeller Produktionen: sie sind sämtlich, ob ,wahr" oder „falsch“, Produkte und zugleich Momente der objektiven Realität, weil sie sämtlich deren ideelle Widerspiegelungen sind. In engerem Sinne bezeichnet "Ideologie“ dann die Formen falscben Bewußtseins, die der objektiv notwendige Widerschein je bestimmter Formationen und Strukturen gesellschaftlicher Wirklichkeit sind; auf sie beziehen sich die Bemühungen marxistischer Ideologieanalyse.

Mit dieser Problematik von Ideologie als falschem Bewußtsein hat es die Widerspiegelungstheorie als materialistische Erkenntnistheorie aber nicht unmittelbar zu tun. Ihre primäre Frage gilt vielmehr den Voraussetzungen der Möglichkeit von Erkenntnis. Diese findet sie einmal - auf der Objektseite - in der objektiven Existenz der Erkenntnisgegenstände, zum anderen - auf der Subjektseite - in der spezifischen Organisation der menschlichen Wahrnehmungsapparatur, der Sinne, vor allem im Zentralnervensystem; es ist übrigens doch beruhigend, wenn selbst die Kritiker nicht daran zweifeln möchten, ,daß der Vorgang der Erkenntnis etwas mit den Nerven zu tun hat" (154), so suffisant sie dies glauben sagen zu müssen. Um nochmals zu verdeutlichen: materialistische Erkenntnistbeorie fragt nach den Voraussetzungen der Möglichkeit richtiger, d.h. adäquater Widerspiegelung der objektiven Realität, also nach der Möglichkeit von Erkenntnis, und sie findet diese Voraussetzungen allgemein eben darin, daß der menschliche Wahrnehmungsapparat, als Moment der objektiven Realität selbst, auf deren adäquate Widerspiegelung abgestellt ist. Die materialistische Ideologieanalyse fragt nach den Quellen der Verzerrung der Widerspiegelung objektiver Realität im Bewußtsein der Menschen und findet sie generell im jeweiligen Entwicklungsstand der Produktivkräfte und den ihnen entsprechenden Organisationsformen der Produktionsverhältnisse. Für die Kritiker ist beides ein- und dasselbe. Sie können zwischen Erkenntnisproblematik und Ideologieproblematik nicht unterscheiden und projizieren diese von ihnen hergestellte Identität auf die Widerspiegelungstheorie.

Irgendwie muß es dąmit zusammenhängen, daß die Kritiker die Behauptung aufstellen, es sei ,die Geschichte der menschlichen Erkenntnis, um die es in der Abbildtheorie ja geht" (160). Natürlich geht es zunächst nicht um die Geschichte der menschlichen Erkenntnis, vielmehr - wir sagten es - um die Voraus setzungen der Möglicbkeit von Erkenntnis, dann allerdings auch um die Bedingun- 
gen ihrer Realisierung. Die Voraussetzungen sind weitgehend überhistorisch; die Bedingungen dagegen verdanken sich geschichtlichen Entwicklungen: Und erst in diesem zweiten Sinne hat es materialistische Erkenntnistheorie dann auch mit der Geschichte der Erkenntnis zu tun (23), die zugleich die Geschichte von Ideologie im allgemeinen also auch die des falschen Bewußtseins ist, und damit ideelles Moment der materiellen Geschichtsprozesses überhaupt. $\mathrm{Zu}$ den beiden bereits erörterten Verschiebungen der Kritiker gesellt sich damit eine dritte: dessen, was Thema der Widerspiegelungstheorie ist (24).

Wir rekapitulieren: Einerseits kreiden die Kritiker der Abbildtheorie an, sie abstrahiere von den je historisch besonderen Formen der Erkenntnis, so daß ihre ,zentralen Sätze auf den Neandertaler und Einstein gleichermaßen passen" (154). (25) Andererseits haben sie sich dafür entschieden, die Abbildtheorie befasse sich mit der Geschichte der menschlichen Erkenntnis: Sie lege eine Theorie der allmählichen Erkenntnismaximierung vor, die die Kritiker als ,naturalistische Anpassungstheorie" glauben diagnostizieren zu können. Danach hätten dann verschiedene Subjekte ,aufgrund des historischen Anpassungsprozesses unterschiedliche Erkenntnisvermögen" (159). Was denn nun? Haben Einstein und der Neandertaler das gleiche Erkenntnisvermögen oder haben sie es nicht? Hier hat die Untersiellungslogik der Kritiker einen Sprung. Entweder abstrahiert die Abbildtheorie von Geschichte, oder sie legt eine, wenn auch noch so finstere, Theorie der geschichtlichen Entwicklung vor.

Die Kritiker sehen hier keinen Widerspruch. Sie entdecken vielmehr, daß die

23 Für eine materialistische Geschichte der menschlichen Erkenntnis vgl. Bernal, J.D., Science in History, London, 1957; deutsch: Wissenschaft in der Geschichte, VEB Deutscher Verlag der Wissenschaften, Berlin (DDR) 1962.

24 ,Die Unfähigkeit der Abbildtheorie besteht darin, daß sie vor lauter Abstraktion die historisch besonderen Erkenntnisformen nicht fassen kann; die Wissenschaft als eine dieser Formen bleibt unerklärt" (154), meinen die Kritiker. Gewiß abstrahiert die Widerspiegelungstheorie als Theorie der Erkenntnisprinzipien von den historisch besonderen Formen der Widerspiegelung. Dagegen dürfte aber auch solange nichts einzuwenden sein, solange die Erörterung der Widerspiegelung auf grundsätzlichem Abstraktionsniveau nicht die Erklärung des historisch Besonderen verhindert. Daß dies durch die Widerspiegelungstheorie geschehe, hätten die Kritiker zeigen müssen, wozu sie nicht einmal ansetzen. Übrigens ist die Formulierung der Kritiker von den ,historisch besonderen Erkenntnisformen", von denen die Wissenschaft eine spezielle sei, interessant. Wollen sie damit die Auffassung andeuten, daß es auch nichtwissenschaftliche Erkenntnis gebe? Sie gerieten damit in bedenkliche Nähe zu abbildtheoretischen Gedankengängen (vgl, oben Anm. 22).

25 Einem Artikel im SPIEGEL, der neuere Forschungsergebnisse der Anthropologie referiert, haben wir entnommen, daß der Neandertaler in der Tat mit Einstein nicht zu vergleichen ist. Er soll nämlich daran zugrunde gegangen sein, daß bei ihm die spezifische organische Fähigkeit zur Sprachentwicklung, also zur Gesellschaftsbildung, noch nicht vorhanden war. Er wird deshalb noch zu den höheren Primaten zu rechnen sein. Erst der Cro-Magnon-Mensch scheint Einsteins Verwandter zu sein: noch gleichzeitig mit dem Neandertaler, überlebte er diesen, weil er sich mit seinen Mit-Cro-Magnon-Menschen intersubjektiv über die objektive Welt verständigen konnte. 
Abbildtheorie geradezu gezwungen sei, sich um eben diese Geschichtstheorie der „,naturalistischen Anpassung“ zu ,erweitern“: sie könne sonst nicht erklären, warum ,das Fallgesetz nicht schon in der Steinzeit entdeckt" wurde, oder warum „die Himmelskörper in Ptolemäus und Aristoteles andere Widerspiegelungen erzeugt (haben) als in Kopernikus, Newton und jedem Oberschüler des 20. Jahrhunderts" (158). Solch bohrende Fragen könnten eben nur dann gegen die Abbildtheorie erfolgreich ins Treffen geführt werden, wenn diese tatsächlich den Nonsens behauptete, alle Formen und Inhalte menschlichen Bewußtseins in Geschichte und Gegenwart stellten schiere Wahrheit dar. Selbst unter solch abwegiger Voraussetzung wäre übrigens immer noch nicht eirzusehen, wieso ,die einzige Geschichtskonzeption, die (immanent gesehen) stimmig mit der Abbildtheorie zu vereinbaren ist, ... eine naturalistische Anpassungstheorie" (159) sein soll (26). Übrigens ist natürlich die Frage legitim, warum das Fallgesetz nicht schon in der Steinzeit entdeckt wurde. Solche Probleme fallen zwar nicht unmittelbar in den Bereich der prinzipiellen erkenntnistheoretischen Fragestellungen der Widerspiegelungstheorie, doch steht ihre Beantwortung keineswegs im Widerspruch zu ihren authentischen Positionen: weil der historische Materialismus nicht im Widerspruch zum dialektischen Materialismus steht.

Das begriffliche Chaos, das die Kritiker anrichten, mitsamt allen Verschiebungen und Entlarvungen, ist nicht willkürlich. Denn noch durch die Widersprüche hindurch bringt der Objektivitätsbegriff der Kritiker, unterstellt als der der Abbildtheorie, System hinein. Als Synonym für Wahrheit und Erkenntnis, abgezogen von allen materiellen Bedingungen ihrer Möglichkeit, lebt er von einer bekannten idealistischen Instanz: dem autonomen Subjekt. Ihrer ideologischen Struktur nach ist diese Instanz, ironischerweise, zugleich. Ursprung der Verhinderung wie Garant der Realisierung von „Objektivität“ und Erkenntnis: Beeinflußt, wie es ist, von gesellschaftlichen Imperativen, Meinungen, Mentalitäten, Vorprägungen aller Art, wird dieses autonome Subjekt einerseits zum Quell alles Falschen, Unwissenschaftlichen, Unwahren; andererseits ist es aber auch der potentielle und einzige Objektivitäts- und Erkenntnisproduzent. Will es objektiv sein, also richtig liegen, muß es sich irgendwie seiner sozialen Vorprägungen entschlagen, sie neutralisieren, zumindest kontrollieren. Dies ist das Thema aller bürgerlichen Ideologiekritik, in der noch die Probleme der klassischen bürgerlichen Erkenntnistheorie soziologisiert nachschwingen (27). Wo, wie bei den Kritikern und etwa Karl Mannheim „Objektivität“ nicht zugleich die Subjektunabhängigkeit der Realität bezeichnet, sondern nur ein Postulat ans forschende Subjekt formuliert und, wenn dieses wissenschaftlich erfüllt ist, ein Ergebnis: „Wahrheit“" meint, dort liegt ein idealistischer Begriff von Objektivität vor. Es ist der der Kritiker und der bürgerlichen Wissenschaft.

Die Behauptung ,immanenter" Stimmigkeit zwischen Abbildtheorie und ,naturalistischer Anpassungstheorie" dürfte aus dem Mißverständnis der Kritiker hinsichtlich des Begriffs der ,relativen Wahrheit" resultieren. Vgl, dazu unter I, 4., sowie Anm. 37 Vgl. zum „Konstitutionproblem“ in der klassischen Philosophie unten Teil II, 3. 


\section{Die Natur der Anpassung und die Anpassung der Natur}

Die Abbildtheorie begreife Erkenntnis (wohlgemerkt: immer im Sinne moderner, in der bürgerlichen Gesellschaft ausgebildeter Wissenschaft) als überhistorische Konstante menschlicher Natur unter Ausklammerung aller gesellschaftlichen Momente einerseits, und sie liefere andererseits eine Historie der Anpassung menschlicher Sinnesorgane an die Natur: das will nun belegt sein. Dazu schicken sich die Kritiker an, indem sie zunächst aus einem Artikel zitieren, der mit den Problemen der Abbildtheorie nichts zu tun hat, ohne daß sie dies kenntlich machten (28). Als handelte es sich um einen Beitrag zur Abbildtheorie, leiten die Kritiker das Zitat mit den Worten ein: „So formulieren auch die'Abbildtheoretiker im Phil. W.B. nachdrücklich und klar ..." (155). Nach diesem einschlägigen Zitat, in dem hauptsächlich die verdächtige Behauptung vorgebracht wird, der Vorgang der Abstraktion scheide das Wesentliche vom Unwesentlichen, fragen die Kritiker ,,nach dem, wovon die Abbildtheorie abstrahiert" und erhalten ,die Antwort: Wichtig ist die Mensch-Natur-Relation, unwichtig die Gesellschaft" (155). Eigentümliche Verkürzung oder charakteristische Verschiebung? Jedenfalls erhält man diese Antwort weder aus dem angefuhrten Zitat, das sich auf Abbildtheorie gar nicht bezieht, noch aus seinem Kontext, noch aus irgendeinem anderen Zitat, das die Kritiker bemühen.

Zum Beleg für ,die Liquidierung des philosophisch-ökonomischen Bewußtseinbegriffs in der Abbildtheorie" wird aus dem Artikel „Bewußtsein" die allgemeinste Definition angeführt, sodann der Satz: „das Bewußtsein entstand als Ergebnis einer langen biologischen Vorgeschichte als Funktion des menschlichen Gehirns ...“ (156, Anm. 9). Hinter den drei Pünktchen der Kritiker verbirgt sich folgende Fortsetzung des Satzes: „welches vor allem unter dem Einfluß der gesellschaftlichen Arbeitstätigkeit seine Entwicklung erfahren hat" (29). Eine sehr eigentümliche Verkürzung. Aber sehen wir weiter, und halten wir uns bloß an die Zitatverwendung, nicht an die begleitenden Reflexionen der Kritiker (30).

Das nächste Zitat stammt, wiederum nicht ausgewiesen, aus dem Artikel „Intersubjektivität“. Zitiert wird:

„Die Intersubjektivität hat ihre Ursache darin, daß die verschiedenen Subjekte etwas Gemeinsames, nämlich die außer ihnen existierende Realität widerspiegeln, deren Eigenschaften und

Der Artikel definiert den intellektuellen Akt der Abstraktion und referiert die verschiedenen Auffassungen von diesem Vorgang in der Denkgeschichte. Von spezifischen Abstraktionsleistungen, die in der Abbildtheorie eine Rolle spielen mögen, ist keine Rede.

29 Phil. W. B. I, S. 197. Wir können dem Leser im übrigen nur empfehlen, den ganzen Artikel „Bewußtsein" durchzusehen, um das Ausmaß der Zitierkunst der Kritiker nachvollziehen zu können.

30 Wir schenken uns also, z. B. folgendes zu analysieren: ,Natürlich würden die Autoren des Phil. W. B. einwenden, sie seien bekannte Marxisten, sie hätten die Bedeutung gesellschaftlicher Momente, insbesondere der Klassen, nie geleugnet ...“ (157). 
Relationen vom Subjekt unabhängig sind, und in der - im Prozeß der Auseinandersetzung des Menschen mit der objektiven Realität entstandenen - relativ identischen Struktur des menschlichen Nervensystems".

Dies legen die Kritiker wie folgt aus:

„So einfach geht das: Obersatz: Gleiche Ursachen erzeugen gleiche Wirkungen. Folge für die Erkenntnistheorie: Eine immer gleiche objektive Realität (,etwas Gemeinsames") und ,relativ gleiche Nervensysteme erzeugen gleiche, für jedermann gültige Widerspiegelungen. Ergebnis: Die Allgemeingültigkeit oder , Intersubjektivität" von Erkenntnis ist zu erklären als Folge gieicher natürlicher Erkenntnisgegenstände und gleicher menschlicher Erkenntnisapparate" (158).

Natürlich sagt das Zitat derlei nicht. Wo findet sich die Formulierung ,immer gleiche objektive Realität" (die Realität ist zwar immer gleich objektiv, keineswegs aber objektiv immer gleicb!), wo ist von gleichen, für jedermann - also alle Menschen in aller Menschheitsgeschichte - gültigen Widerspiegelungen die Rede? Freilich: wenn sich die Menschen über die Beschaffenheit ihrer Welt je und je verständigen konnten und können, so war dafür stets Voraussetzung, daß nicht jeder eine andere Welt mit jeweils besonderen Sinnesorganen rezipierte. Dies sagt das Zitat, und selbst die Kritiker werden das nicht bestreiten wollen. Daß diese Rezeption stets adäquate Widerspiegelung, also Erkenntnis sei, ist dem Zitat dagegen auch bei weitester Auslegung nicht zu entnehmen. Es ist immer dasselbe: einmal der Abbildtheorie unterstellt, ihr sei alles Bewußtsein Erkenntnis, mag man ihr dann auch nachsagen, Intersubjektivität bedeute ihr Wahrheit, es kommt nicht mehr drauf an. Also noch einmal: Intersubjektivität resultiert zwar einerseits aus der Subjektunabhängigkeit der Realität, und andererseits aus der Gleichartigkeit der menschlichen Wahrnehmungsapparate, die diese Realität widerspiegeln und prinzipiell auch darauf angelegt sind, sie adäquat widerzuspiegeln, - doch ist sie selbst nicht Erkenntnis; auch falsches Bewußtsein ist, als objektiv reales, intersubjektiv, und zwar nicht minder wie die adäquate Widerspiegelung der objektiven Realität, die als Erkenntnis objektiv wahr ist. Daß das Phil. W.B. nicht den Unfug behauptet, die Intersubjektivität des gesellschaftlichen Bewußtseins sei gleichbedeutend mit einer überhistorisch allgemein stets gegebenen Realität wissenschaftlicher Erkenntnis, hätten die Kritiker den auf ihr Zitat unmittelbar folgenden Zeilen entnehmen können:

„Aber auch die Unterschiede in Erlebnis-, Wahrnehmungs- und Denkstruktur der Individuen sind letztlich dem Verhältnis des Menschen zur objektiven Realität verdankt, insofern erstens ihre Verschiedenheit auf verschiedene historische Entwicklungsstufen (z.B. der muthologischen Denkweise urgemeinschaftlicher Prägung und der wissenschaftlichen Denkweise unserer Zeit) durch den Grad der Bewältigung der objektiven Realität bestimmt sind, und zweitens die subjektiven Unterschiede im Erleben, Wahrnehmen und Denken in ein und derselben Epoche durch das subjektiv-konkrete Verhältnis zur objektiven Realität, durch den gegebenen Platz im gesellschaftlichen Gefüge (Klassenstandpunkt, Berufsinteresse, Familientradition, gewordene und ererbte Struktur des Nervensystems usw.) bedingt werden, so daß zwar verschiedene Aspekte, aber stets Aspekte der objektiven Realität widergespiegelt werden" (31). 
Unabhängig davon, daß diese - und auch die darauf folgenden - Ausführungen in ihrer verkürzten Form nicht ganz unproblematisch sein mögen, ist festzuhalten: hier wird das Gegenteil dessen gesagt, was die Kritiker ständig zu beweisen suchen. Vor allem dies, daß die Formen der Widerspiegelung in Geschichte und Gegenwart mannigfaltig sind, keineswegs sämtlich den Charakter von Erkenntnis im modernen Sinne aufweisen, vielmehr in ihren jeweiligen Brechungen gesellschaftlich bedingt sind, jedoch darin ihr Gemeinsames haben, daß sie stets objektive - natürliche und gesellschaftliche - Realität widerspiegeln.

Betrachten wir nun den „Nachweis“ der ,naturalistischen Anpassungstheorie". Die Kritiker verweisen auf die Ausführlichkeit ihres Zitierens - wir wollen sehen, ob es dadurch gediegener wird. Das erste Zitat stammt aus dem Artikel „Erkennbarkeit der Welt"“, das zweite aus dem Artikel „Sinne“. Darauf, daß das Zitat aus ,Erkennbarkeit der Welt" nicht die Auffassung der Autoren des Phil. W.B. wiedergibt, sondern die kybernetische Sichtweise des Erkenntnisprozesses - von der allerdings gesagt wird, daß sie, ebenso wie die Begriffe der Informationstheorie, dazu beitrage, „manche erkenntnistheoretische Sachverhalte begrifflich zu präzisieren" - sei nur nebenbei hingewiesen. Das zweite Zitat nun schildert die Entstehung der Sinnesorgane als „Anpassung an die für das Leben der Organismen wichtigsten Eigenschaften der objektiven Realität". Den Kritikern zufolge zeigen die zitierten Sätze, „zu welcher Vorstellung von Geschichte die Abbildtheorie drängt" (159). Wirklich: eine Geschichtsvorstellung, die die Menscbbeitsgescbicbte begriffe als allmählichen, evolutionistischen Herausbildungsprozeß der Sinnesorgane durch Anpassung an die Natur, wäre nicht nur ,eine ungeheuer triviale und falsche Geschichtserklärung" (158), sie wäre Nonsens, und im Gegensatz zu den Kritikern könnten wir uns selbst ,,unter Abstraktion von allem Gesellschaftlichen den Prozeß der Menschheitsentwicklung“ (soll hier wohl heißen: Menschheitsgescbicbte) nicht „als einen Vorgang allmählicher Anpassung vorstellen“ (159). Derlei hat unseres Wissens zuletzt Gottfried Benn aufgrund biologisch-ideologischer Mythen literarisch frappierend vertreten; die evolutionistische „Verhirnung“ des Menschen, die „,progressive Cerebration“ war ihm, der zum Urschleim strebte, allerdings Verfallsgeschichte (32), während die Variante von Abbildtheorie, die die Kritiker entwerfen, eine solche Historie ja wohl für fortschrittlich hielte.

In ihrem Eifer, der Abbildtheorie die Behauptung nachzuweisen, die Menschheitsgeschichte sei umstandslos bloße Naturgeschichte, nehmen nun unsere Kritiker eine besonders eigentümliche Verkürzung vor: sie tun so, als beziehe sich das Zitat auf die Menschheitsgeschichte. Es bezieht sich aber auf die Naturgeschichte vor dem Auftreten des Menschen, der selbst mit seinen hochkomplizierten Sinnesapparaten als Ergebnis eines evolutionär-biologischen Entwicklungsprozesses dargestellt wird.

Vgl. Peter Reichel, Künstlermoral. Das Formalismus-Programm spätbürgerlicher Dichtung in Gottfried Benns ,Gereimter Weltanschauung", Berlin (DDR) 1974, Reihe: Zur Kritik der bürgerlichen Ideologie, 42. bes. S. $43 \mathrm{ff}$ : „Biologistische Regression und Gesellschaftsprognose". 
Auf die davon substantiell unterschiedenen Spezifika und Strukturen der Menschheitsgeschichte und ihre, gesellschaftlich vermittelten, Auswirkungen auf die Differenzierung der menschlichen Sinne geht das Wörterbuch nunmehr nach einem Absatz ein, der eine genaue Scheidung zwischen der Behandlung der Naturentwicklung und der der Menschheitsgeschichte vornimmt. Vielleicht haben die Kritiker das nicht mehr gelesen (33).

Während sie ständig behaupten, die Abbildtheorie verbreite das „Geschichtsbild einer sukzessiven Höherentwicklung von ,Organismen ${ }^{6}$ (160), sie sehe nur „fließende Übergänge zwischen unbelebter und belebter Natur, zwischen Tier und Mensch und weiterhin innerhalb der eigentlichen Menschheitsgeschichte" (159, Anm. 16; Zusatz der Kritiker: ,alles s.o. Zitate“, also jene, die wir uns gerade angesehen haben!), setzt die materialistische Erkenntnistheorie, auch ihre Darstellung im Phil. W.B., stets eine scharfe Zäsur zwischen der biologischen Evolution vor dem Auftreten des Menschen und dem über Arbeit und Sprache, also gesellschaftlich vermittelten Prozeß der Bewußtseinsbildung und ihrer Formen in der Menschheitsgeschichte (34).

Wir können hier diese Fragen nicht in der Sache selbst erörtern. Um zur Information über das abbildtheoretisch Selbstverständliche jedoch noch ein Weiteres zu tun, zitieren wir aus dem schon angeführten Standardwerk von Todor Pawlow:

„Die Darwinsche Evolutionstheorie (natürliche Auslese, Anpassung, Vererbung usw.) kann weder das Erscheinen des Menschen noch jenen Unterschied erklären, der zwischen ihm und dem Tier besteht. Der Mensch als solcher, d.h. als neuer Typ eines handeinden und denkenden Subjekts, ist kein Produkt einer lediglich natürlichen Auslese. Hier endet der Darwinismus und beginnt der historische Materialismus von Marx und Engels, der diese Frage nicht naturalistisch, sondern sozial-historisch löst". Und weiter, nachdem die Arbeit, die Sprache, die gesellschaftliche Organisation als wesentlich neue Momente angeführt wurden: ,,Mit einem Wort: all dies bringt ein wirklich menschliches, d.h. ein qualitativ neues gesellschaftlich-historisches und psycho-physiologisches Subjekt des Denkens und Handelns hervor. Und deshalb spricht Marx davon, daß sogar die Sinnesorgane des Menschen Produkte einer gesellschaftlich-historischen Entwicklung darstellen" (35).

Man mag ja am „Sowjet-Marxismus“ allerlei kritisieren können - das ist hier nicht unser Thema -: gegen Anwürfe wie die der Kritiker ist er jedenfalls gefeit. Oder

Aus Platzgründen verzichten wir auf die Wiedergabe dieses Absatzes im Phil. W. B. und bitten den Leser, dort selbst nachzulesen (II, S. 983). Wir machen dabei besonders auf ein längeres Marx-Zitat aufmerksam, das den Kern der Ausführungen des Phil. W. B. an dieser Stelle darstellt und aus den ,ökonomisch-philosophischen Manuskripten" stammt, (MEW, Erg. 1, S. 541), wo man überprüfen kann, daß Marx die Grundfragen der Erkenntnistheorie für sich bereits zu Beginn seiner theoretischen Arbeit widerspiegelungstheoretisch beantwortet hat.

34 Vgl. dazu Ausführungen im Artikel ,,Bewußtsein“ des Phil. W. B., die die Kritiker unterschlagen, sowie den ganzen Artikel „Gesellschaftliches Bewußtsein“, den die Kritiker gar nicht erst heranziehen.

35 Pawlow, a.a.O., S. 145, Hervorhebung im Original. Pawlow bezieht sich in der Schlußbemerkung des Zitats auf jene Ausführungen von Marx, auf die wir in Anm. 33 hingewiesen haben und die im Phil. W. B. zitiert werden. 
muß ernsthaft in Erinnerung gerufen werden, daß eine seiner kardinalen Unterscheidungen die zwischen dialektischem Materialismus und historischem Materialismus, zwischen Naturdialektik und historisch-gesellschaftlicher Dialektik ist? (36) Und daß diese Unterscheidung, was immer für Probleme sie in sich bergen mag, doch wohl jedenfalls dem Tatbestand Rechnung trägt und zum Ausdruck bringt, daß mit dem Menschen, obgleich natürlich auch er Naturprodukt, Materie ist, einschließlich seines Bewußtseins und dessen Formen, der Naturprozeß durch Erscheinen eines handelnden und denkenden Subjekts eine qualitative Änderung erfährt? Daß hier nicht mehr bloß Organismen der "Natur" sich anpassen, der sie selbst materiell zugehören, sondern die Natur ihrerseits den Bedürfnissen eines Organismus, des menschlichen, angepaßt wird durch menschliche, gesellschaftliche Praxis, wobei sich die Differenziertheit dieses Organismus historisch entfaltet?

36 Als ,merkwürdiges Faktum" bezeichnen die Kritiker, „daß die Abbildtheorie - im direkten Gegensatz zur Marx'schen Kritik der Ökonomie ... - bislang von bürgerlicher Kritik unbehelligt blieb" (156, Anm. 8), und das komme eben davon, daß die Bürger an dieser bürgerlichen Theorie nichts auszusetzen haben (wieso ist es dann übrigens ein „merkwürdiges“ Faktum?). Dies ist wohl so ziemlich das stärkste, was die Kritiker sich geleistet haben. Schließlich wurden die antikommunistischen ,Widerlegungen" des "Diamat" von Autoren wie Wetter, Borkenau, Bochenski, Brzesinski bis hin zu Fetscher nicht in hermetischen Seminarzirkeln betrieben, sondern über die Landeszentralen für Politische Bildung verteilt. Erstaunlich, wenn die Kritiker den Auswirkungen dieser „Markt-Selektion“ entgangen sein sollten. Aber sie sind es ja auch gar nicht! Im Prinzip, wenn auch in revolutionär-, marxistischer" Drapierung, reproduzieren sie nämlich eben jene bürgerliche Kritik, die es angeblich nicht gibt: man lese einmal etwa den Artikel "Widerspiegelung" von N. Lobkowicz in der Enzyklopädie „Sowjetsystem und demokratische Gesellschaft", Bd. 6, S. 951, oder in dem Büchlein „Der Sowjetrussische Dialektische Materialismus (Diamat)", München 1950, von I. M. Bochenski das Kapitel „Der Realismus und der Rationalismus", S. $88 \mathrm{ff}$.. Ubrigens sind diese Autoren redlicher als die Kritiker: nicht nur referieren sie die Positionen der Abbildtheorie wenigstens halbwegs angemessen, sie kritisieren sie auch im Rahmen eines Frontalangriffs auf den Materialismus, während die Kritiker zwar das gleiche tun, aber vorgeben, nur ein Einzeltheorem kritisch zu prüfen. - Die Kompendien antikommunistischer ,Widerlegungen" des „Diamat" sind oft von Jesuiten verfaßt, die, ihrer thomistischen Ausbildung gemäß, die materialistische Erkenntnistheorie gewöhnlich als ,naiven Realismus" kennzeichnen, wohl, um die Differenz zum heiligen Realismus des Thomas v. Aquin gebührend gegen den des Lenin abzuheben. Andererseits - das scheint wieder für die Meinung der Kritiker zu sprechen - setzt sich die moderne bürgerliche Fachphilosophie sprachanalytischer, neorealistischer oder kritisch-rationalistischer Provenienz in der Tat mit der marxistischen Erkenntnistheorie nicht auseinander. Das macht aber: sie nimmt überhaupt nichts, was mit materialistischer Wissenschaft zusammenhängt, zur Kenntnis - womit sie zwar ihre Irrelevanz, aber kaum einen bürgerlichen Charakter der Widerspiegelungstheorie beweist. Es gibt allerdings, von Platon bis Wittgenstein, auch nicht-materialistische Varianten von Abbildtheorie. Es kommt immer darauf an: Abbild wovon, Widerspiegelung wessen? Wem diese Frage unerheblich ist, der kann freilich leicht die materialistische Abbildtheorie für bürgerlich halten. 


\section{Die Wahrheit der ,,relativen Wahrheit ${ }^{66}$}

Der Aufweis einer der Geschichtsvorstellung ,, nahe verwandten“ (160) relativistischen Wahrheitstheorie der Abbildtheoretiker ist ein weiteres Anliegen der Kritiker. Nahe verwandt ist sie allerdings: insofern sie nämlich das Pendant der ,,naturalistischen Anpassungstheorie" und wie diese freihändiges Konstrukt der Kritiker ist. Logisch: wenn die Menschheitsgeschichte die der Anpassung von Rezeptionsorganen an die Natur ist, so ist sie zugleich linear und kontinuierlich verlaufende Erkenntnisgeschichte. Es zeichnet sich dann das positivistische Bild eines historischen Ablaufs $a b$, in dem ,, beständig unzutreffende Vorstellungen ... durch Vorstellungen ersetzt werden, die etwas weniger unzutreffend ... sind" (162).

Diese Vorstellung - sehr entfernt mag sie vielleicht an wissenschaftsgeschichtliche Auffassungen etwa Poppers erinnern - erweist sich den Kritikern als die der Abbildtheorie, weil in ihr der Begriff der, relativen Wahrheit“ eine Rolle spielt. Zwar, so die Kritiker, sei der Begriff der relativen Wahrheit ,absurd“ (162), weil es eine ,ein bißchen falsche" Wahrheit nicht geben könne (162), doch in die Logik der Abbildtheorie gehöre genau diese unhistorische ,Körnchen-, Tröpfchen- oder

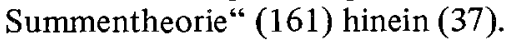

Die Abbildtheorie kann zwischen wahr und falsch nicht unterscheiden, hat ,überhaupt keinen Begriff von exakter wissenschaftlicher Erkenntnis" (162), huldigt also einem haltlosen Relativismus: diese verblüffende Enthüllung der Kritiker offenbart ihr Geheimnis dem, der bei Lenin nachliest (38) oder auch nur

Im Zusammenhang der Problematik von relativer und absoluter Wahrheit reproduziert sich jene Unvereinbarkeit zweier Vorwürfe der Kritiker, auf die wir schon hingewiesen haben. Entweder soll die Abbildtheorie einen endlosen Relativismus, wonach nie etwas wirklich wahr sein kann, vertreten, oder die ,immergleiche objektive Form" (161) der Erkenntnis behaupten. Entweder immergleich oder immer ungleich. Entweder promoviert die Abbildtheorie alles, was Menschen je sich vorgestellt und gedacht haben, zur Erkenntnis im Sinne moderner Wissenschaft; ,, addiert" sie ,,wissenschaftliche, vorwissenschaftliche und nichtwissenschaftliche Erkenntnisse": dann kann sie der Vorwurf nicht treffen, daß etwa die ,magischen Vorstellungen früher Epochen . . . sich in keiner Weise wissenschaftlichen Erkenntnissen hinzuzählen lassen“, weil diese „nicht relativ wahr, sondern objektiv falsch" seien (161, Anm. 19); denn sie hat ja gar kein Kriterium, wahr und falsch zu unterscheiden. Oder sie soll stets gleiche Wahrheitselemente, alle gleich objektiv im Sinne von ,wahr", zu einer „Erkenntnisgeschichte ohne Brüche“ addieren und behaupten, die ,objektive Form von Erkenntnis sei die Naturform von Erkenntnis“ (161): dann würde sie den Schwachsinn vertreten, etwa die magischen Vorstellungen früher Epochen seien gleichermaßen ,wahr" wie die Erkenntnisse moderner Naturwissenschaft und könnten diesen hinzugezählt werden; sie könnten dann auch nicht etwas we niger wahr sein.

38 Lenin, Werke 14 (Materialismus und Empiriokritizismus), Kap. II, 5 : Absolute und relative Wahrheit ..., S. 126 ff. 
die nicht-zitierten Passagen aus dem Artikel ,Wahrheit“ im Phil. W.B. (39). Solche Lektüre belehrt nämlich dahin, daß der Begriff „,relative Wahrheit" im Zusammenhang der marxistischen Erkenntnistheorie eine Bedeutung hat, von der die Kritiker offenkundig nichts ahnen. Sie halten ihren eigenen Alltagsbegriff von „relativ" so, wie man etwa davon sprechen mag, man sei relativ, also ein bißchen, müde - für den der Abbildtheorie. Was die Kritiker schon mit ihren Begriffsverständnis von „objektiv" gezeigt haben, wiederholen sie nun an der Bedeutung von ,relativ“. Heißt ihnen „objektiv" einzig soviel wie „wahr", so bedeutet ihnen ,relativ" eine Minderung des Vollkommenen, die, auf Wahrheit bezogen, nur deren Vernichtung sein kann: „relativ wahr" ist „falsch“. Sowenig aber der Objektivitätsbegriff der Abbildtheorie sich reduzieren läßt auf einen modernen Begriff von wissenschaftlicher Erkenntnis, sowenig bedeutet der abbildtheoretische Begriff des „Relativen“6 eine Minderung des für wahr Erkannten und Erwiesenen in Richtung aufs Ungefähre. Worum handelt es sich also tatsächlich?

Gegen eine Vermengung zweier Probleme bei Bogdanow richtet Lenin folgende Doppelfrage:

„1. Gibt es eine objektive Wahrheit, d.h. kann es in den menschlichen Vorstellungen einen Inhalt geben, der vom Subjekt unabhängig ist, der weder vom Menschen, noch von der Menschheit abhängig ist? 2. Wenn ja, können dann die menschlichen Vorstellungen, die die objektive Wahrheit ausdrücken, sie auf einmal, vollständig, unbedingt, absolut oder nur annähernd, relativ ausdrücken? Diese zweite Frage ist die Frage nach dem Verhältnis zwischen absoluter und relativer Wahrheit" (40).

Das Wörtchen „annähernd“ - das ist der Irrtum der Kritiker - bezieht sich nicht auf einen Grad an Wahrheit, einen Grad an Richtigkeit von Wissen, sondern auf den Grad seiner Teilhabe an der ganzen Wahrheit, einer abschlußhaft vollständigen. Relativ ist eine Wahrheit nicht insofern, als irgend etwas an ihr falscb wäre, sondern

„die Grenzen der Wahrheir jedes wissenschaftlichen Satzes sind relativ und können durch die weitere Entwicklung des Wissens entweder weiter oder enger gezogen werden" (41).

39 Also den ganzen Artikel: denn die beiden Zitate, die die Kritiker daraus bringen, beweisen ihre Unterstellungen in keiner Weise (wir verzichten jetzt darauf, dies im einzelnen zu zeigen), sind nur aus dem nicht-zitierten Zusammenhang verständlich und zudem weiderum ,eigentümlich verkürzt ${ }^{t}$ (im ersten $Z$ itat ist eigentümlicherweise die Stelle „infolge ihrer Abhängigkeit von den jeweiligen Erkenntnisbedingungen Elemente des Relativen enthält" durch Pünktchen ersetzt; im zweiten, ein Lenin-Zitat, fehlt der zweite Teil, der den ersten erst vorständlich macht; vgl. dazu unten Anm. 41).

40 Lenin, Werke 14, S. 116. Dieses Zitat wird im Phil. W.B. unmittelbar vor der von den Kritikern herangezogenen Stelle angeführt. - Bedenkt man an diesem Zitat Lenins Begriffsverwendung von ,objektiv", so ergibt sich folgendes: einerseits gebraucht er hier „objektiv" als Attribut zu „Wahrheit", also umgangssprachlich. Zugleich bestimmt er diesen Sprachgebrauch dahin: „objektiv" ist die adäquate Widerspiegelung der vom Subjekt unabhängigen Realität - und das ist der systematische Punkt, um den es geht. Die ganze Problematik liegt in der genauen Reflexion der Begtiffspaare ,objektiv subjektiv" und ,absolut-relativ".

41 Lenin, Werke 14, S. 129. Dies ist die unzitierte zweite Hälfte des von den Kritikern aus dem Phil. W.B. herangezogenen Lenin-Zitats! 
Der Gegensatz von „relativ" ist also nicht „objektiv“", sondern „,absolut". Eine gewisse Schwierigkeit für das Verständnis dieses Zusammenhangs von relativer und absoluter Wahrheit mag sich für die Kritiker wiederum daraus ergeben, daß es ein dialektischer Zusammenhang ist. In einer Hinsicht muß man nämlich sagen, daß Wahrheit immer relativ und nie absolut ist; in anderer Hinsicht, daß Wahrheit immer zugleich relativ und absolut ist. Immer relativ und nie absolut ist Wahrheit, soweit man unter absoluter Wahrheit idealistisch die Erkenntnis des Ganzen ,,auf einmal, vollständig, absolut" oder als Schlußsumme der Erkenntnis alles Erkennbaren versteht. Gegen diese Vorstellung wendet Engels ein, daß

„ein allumfassendes, ein für allemal abschließendes System der Erkenntnis von Natur und Geschichte ... im Widerspruch (steht) mit den Grundgesetzen des dialektischen Denkens, was indes keineswegs ausschließt, sondern im Gegenteil einschließt, daß die systematische Erkenntnis der gesamten äußeren Welt von Geschlecht zu Geschlecht Riesenfortschritte machen kann" (42).

Insofern alles zu Erkennende dialektisch Veränderungen unterworfen ist und alle Erkenntnis Prozeß ist, ist der Gedanke einer solchen absoluten Wahrheit historischmaterialistisch gar nicht zu fassen, Wahrheit also immer relativ. Andererseits ist sie zugleich auch stets absolut, insoweit sie zwar nie umfassend, vollständig und abschlußhaft, nichts desto weniger aber unbedingt wahr ist.

Hinsichtlich ihrer Grenzen also ist jede Erkenntnis prinzipiell relativ wahr. Da sie aber wahr ist, und soweit sie dies ist, ist sie es innerhalb dieser Grenzen absolut, ist sie kein Quentchen, kein bißchen falsch. Die je konkrete Schwierigkeit ist, daß die Grenzen einer Wahrheit oft enger sind, als man dachte, und manchmal weiter als man annahm. Der Fortschritt im Erkanntnisprozeß besteht darin, solche Grenzziehungen zu präzisieren, d.h. die Reichweite einer relativen Wahrheit abzustecken, ohne ihre Relativität je aufheben zu können. Gesellschaftliche Praxis im weitesten Sinne des Wortes ist das Medium, durch das hindurch sich das Erkenntnisobjekt verändert, der Erkenntnisprozeß vollzieht und die Grenzen der Erkenntnisse erweitern (43).

Engels, MEW 19, S. 206 f. Die Stelle wird im Artikel „,Wahrheit“ des Phil. W.B. zitiert.

Es ist im vorliegenden Zusammenhang, der eine positive Darstellung der Probleme der Abbildtheorie nicht leisten kann, sondern nur dem Aufweis der Haltlosigkeit der Kritiker-Invektiven dient, leider nicht möglich, auf das für die materialistische Erkenntnistheorie zentrale Problem der Praxis als Kriterium der Wahrheit einzugehen, das schon in Marx' zweiter These über Feuerbach (MEW 3, S. 533) deutlich wird. Die Kritiker, für die sich diese wesentliche Frage natürlich überhaupt nicht stellt, versuchen lediglich, aus dem Artikel „Praxis“ des Phil. W.B. eine Belegstelle für ,extremen Relativismus“ herauszuziehen (162, Anm. 20). Auch dies ,gelingt" nur durch eine weitere eigentiimliche Verkürzung, die wir Fälschung nennen müssen. Der nächste Satz im Phil. W.B. nach der von den Kritikern herangezogenen Stelle lautet nämlich: „Analoges gilt für eine als falsch nachgewiesene Aussage“. Den Kritikern zufolge dürfte es als falsch nachgewiesene Aussagen abbildtheoretisch aber gar nicht geben. 
Der materialistisch-dialektische Grundgedanke der Unterscheidung von relativer und absoluter Wahrheit ist - und deshalb wollen wir unsere knappen Anmerkungen zu dieser grundlegenden, von den Kritikern abgrundtief unverstandenen Frage damit beschließen - wohl kaum deutlicher herausgearbeitet worden als in folgenden Worten Lenins:

„Vom Standpunkt des modernen Materialismus, d.h. des Marxismus, sind die Grenzen der Annäherung unserer Kenntnisse an die objektive, absolute Wahrheit geschichtlich bedingt, unbedingt aber ist die Existenz dieser Wahrheit selbst, unbedingt ist, daß wir uns ihr nähern. Geschichtlich bedingt sind die Konturen des Bildes, unbedingt aber ist, daß dieses Bild ein objektiv existierendes Modell wiedergibt. Geschichtlich bedingt ist, wann und unter welchen Umständen wir in unserer Erkenntnis des Wesens der Dinge bis zu der Entdeckung des Alizarins im Kohlenteer oder bis zur Entdeckung der Elektronen im Atom gelangt sind, unbedingt aber ist, daß jede solche Entdeckung ein Schritt vorwärts auf dem Wege der ,unbedingt objektiven Erkenntnis' ist. Kurzum, geschichtlich bedingt ist jede Ideologie, unbedingt aber ist, daß jeder wissenschaftlichen Ideologie (zum Unterschied beispielsweise von der religiösen) die objektive Wahrheit, die absolute Natur entspricht. Ihr werdet sagen: Diese Unterscheidung zwischen relativer und absoluter Wahrheit ist unbestimmt. Ich antworte darauf: Sie ist gerade ,unbestimmt' genug, um die Verwandlung der Wissenschaft in ein Dogma im schlechten Sinne des Wortes, d.h. in etwas Totes, Erstarrtes, Verknöchertes zu verhindern, sie ist aber zugleich ,bestimmt' genug, um sich auf das entschiedenste und unwiderruflichste vom Fideismus und vom Agnostizismus, vom philosophischen Idealismus und von der Sophistik der Anhänger Humes und Kants abzugrenzen. Hier ist die Trennungslinie, die ihr nicht bemerkt habt, und weil ihr sie nicht bemerkt habt, seid ihr in den Sumpf der reaktionären Philosophie hinabgeglitten. Dies ist die Trennungslinie zwischen dialektischem Materialismus und Relativismus" (44).

\section{Die Erkenntnistheorie der Kritiker}

Nachdem wir Argumentierweise und Art der Zitatverwendung bei den Kritikern beleuchtet haben, wollen wir nun herausfinden, welche Vorstellungen sie von einer Erkenntnistheorie haben, die nach ihrer Ansicht den Namen marxistischer Wissenschaftlichkeit verdient. Da die Kritiker diese Vorstellungen nicht explizieren, sind wir in diesem Teil unserer Anmerkungen gezwungen, selbst das Terrain von Vermutungen und Interpretationen zu betreten, für die sich jedoch genügend Hinweise terminologischer und argumentativer Art im Text der Kritiker finden. Anhand einer Analyse vor allem der Einleitung ihres Aufsatzes wollen wir zunächst das Geschichtsbild der Kritiker charakterisieren und die damit zusammenhängende Erwar-

44 Lenin, Werke 14, S. $130 \mathrm{ff}$. Auch hier osziliert übrigens der Gebrauch des Begriffs ,,objektiv“; vgl. dazu oben Anm. 40. Im übrigen hoffen wir, daß dem Leser nunmehr das ganze Ausmaß der Witzigkeit deutlich geworden ist, die in der kritischen Frage liegt: "Trifft es wirklich zu, wenn man behauptet, die Winkelsumme im Dreieck betrage möglicherweise 179 Grad? " (162). Tief betroffen geht der Abbildtheoretiker in sich, bekennt sich unter allen Umständen für die euklidische Geometrie und erklärt die Behauptung, daß im Unendlichen sich die Parallelen schneiden, für relativistischen Wahnsinn. 
tung an Erkenntnistheorie. Es folgen sodann einige Bemerkungen zur Bedeutung von Erkenntnistheorie innerhalb der materialistischen Wissenschaft. Abschließend wollen wir dann versuchen, die Struktur jenes Bewußtseins herauszuarbeiten, daß eine Kritik wie die von uns untersuchte und ihre theoretisch-politischen Implikationen bedingt und ermöglicht. Dabei kann es sich freilich nur um eine skizzenhafte, unvollständige und vorläufige Analyse des Phänomens handeln.

\section{Geschichtsbild und Erwartung an Erkenntnistheorie}

„Seit den Krisen der zwanziger Jahre, spätestens aber seit Hiroshima und dem Fall Oppenheimer", so beginnen die Kritiker, sei „die einst mit der Wissenschaft verbundende Fortschrittsideologie erschüttert" worden. Frühere Hoffnungen haben sich „als Illusion“ entpuppt. Auch „im Bewußtsein der Massen“ erscheinen „Wissenschaft und ihre Anwendung als Technik ... heute... mehr und mehr als bedrohliche Machwerke, der gesellschaftliche Zustand, den sie nach sich ziehen, als Unheil und Verderben“" (151).

Von wessen Fortschrittsideologie mag hier die Rede sein? Und, gesetzt, wir wuißten es, wie kommt es zur Terminwahl ihrer Erschütterung? Nehmen wir einmal an, die Kritiker meinen die Fortschrittsideologie der Bourgeoisie, dann erscheinen die Zeitpunkte mindestens etwas willkürlich gewählt. Bekanntlich läßt die Fortschrittsideologie der Bourgeoisie immer dann und dort an Intensität nach, wenn ihre fortschrittliche Rolle in der Geschichte erfüllt ist, oder wo sie einsehen muß, „daß alle Bildungsmittel, die sie erzeugt, gegen ihre eigene Zivilisation rebellierten, daß alle Götter, die sie geschaffen, von ihr abgefallen waren", wie Marx für die französische Bourgeoisie zu Beginn der zweiten Hälfte des vorigen Jahrhunderts bemerkte (45). Für die englische Bourgeoisie fällt dieser Zeitpunkt dagegen bereits mit dem Abschluß der industriellen Revolution zusammen und dem ersten, nicht mehr dem Zufall zuzuschreibenden Auftreten der Überproduktionskrisen (46). Die deutsche Bourgeoisie hat wiederum so einen rechten Fortschrittsoptimismus eigentlich nie entwickelt (47). Jedenfalls war längst vor dem Ersten Weltkrieg allenthalben

Marx, Der 18. Brumaire des Louis Bonaparte, MEW 8, S. 153

46 Vgl. z. B. Marx, Kapital I, MEW 23, S. 20 ff. Ausdruck des daraus resultierenden Verlust an Fortschrittsoptimismus war z. B. die Malthus'sche Bevölkerungstheorie, deren Kern bekanntlich darin besteht, aus den Wirkungen der Gesetze des kapitalistischen Akkulumationszyklus auf dem Beschäftigungsgrad der Arbeiterklasse (industrielle Reservearmee) absurde Thesen über das natürliche Verhältnis von Bevölkerungszunahme und Lebensmittelproduktion, damit über das Schicksal der Menschheit schlechthin zu entwickeln.

47 Die wesentlichen Strömungen der deutschen bürgerlichen Intelligenz, die das Bewußtsein ihrer Klasse artikulierte, sind immer noch am besten bei Georg Lukàcs, die Zerstörung der Vernunft, Werke 9, Neuwied/Berlin 1962 zu studieren, wenn man nicht die Originale heranziehen will. 
Mode der bürgerlichen Intelligenz geworden, den Fortschritt als Illusion zu entlarven, (48) und die Struktur dieser Kassandrarufe hat sich bis zu den jüngsten Verlautbarungen des Club of Rome nicht geändert.

Insoweit bleibt dunkel, weshalb nun die Krisen der zwanziger Jahre bzw. dann der Fall Oppenheimer in diesem Sinne etwas Neues erbracht hätten und einen besonderen Tiefpunkt bourgeoisen Optimismus darstellen sollen. Es sei denn gemeint, solche Niedergeschlagenheit resultierte aus den Hoffnungen der Arbeiterklasse und ihrer Verbündeten in den Mittelklassen und der Intelligenz, die sich an den Sieg der Oktoberrevolution knüpften. Aber daran denken die Kritiker wohl kaum, denn gerade bei „den Massen“" soll die Fortschrittsgläubigkeit seither besonders gelitten haben. Das zeigt sich den Kritikern merkwürdigerweise an ,zeitgemäßen Kindercomics und (den) aufs Massenbewußtsein zielenden Produkten der Filmindustrie“, also nicht an Ausdrucksformen ,des Massenbewußtseins“ selbst, sondern an bourgeoisen Medien seiner Manipulation; schließlich an Orwell und Huxley, die ja den bürgerlichen Salonkritikern von Kultur und Zivilisation so zusammengehörig scheinen wie Goethe und Schiller einem deutschen Studienrat, nur weil beide sich gelegentlich des gleichen literarischen Genres, der negativen Utopie, bedient la ben (49).

Obwohl sie es in der dann folgenden Kritik der Abbildtheorie so vehement prätendieren, denken die Kritiker keineswegs in den Kategorien der marxistischen Analyse kapital istischer Klassengesellschaft, deren Entwicklungsstadien zur Analyse von Bewußtseinsformen herangezogen würden. Sie reproduzieren vielmehr bloß die Klischees bürgerlicher Kulturkritik, die allerdings linke Varianten hat. Nicht das Bewußtsein von Klassen steht infrage, sondern das ,,der Massen“. Nicht die Produktionsverhältnisse sind es, die den Fortschritt aufhalten und die Katastrophe denkbar machen - das Unheil bergen die Produktionsmittel, die die bürgerliche Gesellschaft entfaltet hat, selbst in sich. Nicht die Bourgeoisie ist inzwischen international, aber national zu jeweils verschiedenen Zeitpunkten, an die Grenzen ihrer progressiven Rolle in der Geschichte geraten, sondern ein Zeitalter ist zu Ende gegangen: das des Fortschrittsglaubens. Die Menscbbeit hat sich in Illusionen gewiegt, die den Katastrophen der Gegenwart - seit den zwanziger Jahren - nicht mehr standhalten

48 Aus einer breiten einschlägigen Literatur, sozusagen als Zitat: George Sorel, Les illusions du progrès, Paris 1908. Sorel verstand sich übrigens als Marxist, der den Sozialismus mit Hilfe des "Mythos" befördern wollte, nachdem sich s. E. der rationale „Fortschrittsglaube" als dazu untauglich erwiesen hatte.

49 Im übrigen hatten sie nichts miteinander zu tun. Während Orwell, aktiver Kämpfer im Spanischen Bürgerkrieg, in ,,1984" seine Erfahrungen mit dem Stalinismus verarbeitete, was mit einem Verlust an Glauben an die Wissenschaft nichts zu tun hat (leider ist er dadurch zum unfreiwilligen Zeugen höchstwissenschaftlicher Totalitarismus-Theorien geworden), gehört Huxley allerdings in das Lager einer skurril-radikalen, christlichkonservativen Zivilisationskritik, deren linke Entsprechungen sich etwa im Umkreis der Frankfurter Schule finden. Wie weit seine "Brave new world" dort goutiert werden konnte, ist nachzulesen bei Th. W. Adorno, Aldous Huxley und die Utopie, in: ders., Prismen, Frankfurt/Main 1955. 
konnten. Es hat sich etwas berausgestellt: denn nie war der Fortschrittsglaube berechtigt, er war nur noch nicht ernüchtert worden. Die „Dialektik der Aufklärung" $(50)$ hat zugeschlagen, die Wissenschaft des bürgerlichen Zeitalters und ihr auf technische Beherrschbarkeit der Natur gerichtetes Denken war menschheitlich ein Irrtum, ein falscher Entwurf, eine Sackgasse: das Ganze war falsch, und das ganz Andere (51) muß gedacht und gewagt werden. Und eben dabei nun hat marxistische Erkenntnistheorie, wie die Kritiker sie meinen, ihre zentrale Aufgabe: sie soll ,das etablierte System der bürgerlichen Wissenschaften von seiner Wurzel her ${ }^{\text {"6 }}$, nämlich als falschen Entwurf, verständlich machen, sie soll ,,die herrschende bürgerliche Wissenschaft und ihre technische Anwendung", transzendieren" (Hervorhebung d. Verf.) und „Möglichkeiten zu ihrer Überwindung“" zeigen (151 f).

Sorge der Kritiker ist nicht, wie gesellschaftliche Verhältnisse herzustellen wären, die es ermöglichen, daß die in der bürgerlichen Gesellschaft entwickelte Wissenschaft nicht mehr destruktiv angewandt werden kann, sondern jene Hoffnungen erfüllen hilft, die einmal auch die aufsteigende Bourgeoisie teilte (52). Ihnen geht es vielmehr ums - zunächst mentale - „Transzendieren“ des „bürgerlichen“ Begriffs von Wissenschaft und der technischen Anwendung seiner Ergebnisse selbst. Dies ist die „Erwartung“ an marxistischer Erkenntnistheorie, die die Kritiker allenthalben keimen sehen und die sie selbst teilen, eine Erwartung, die eben jene marxistische Erkenntnistheorie, die sich als Abbildtheorie darstellt, nicht erfüllt. Nicht erfüllen kann, weil sie, selbst dem ,bürgerlichen“ Begriff von Wissenschaft verhaftet, auch nur in bürgerlichen Gesellschaftsformen auftreten kann: „Sie destruiert nicht, sondern festigt die bürgerliche Wissenschaftsauffassung" (152; im Original hervorgehoben) (53).

Solche Erwartung an Erkenntnistheorie resultiert 2us dem skizzierten Geschichtsbild. Politisch mündet sie in jenen „Anti-Revisionismus“, der in dem

Max Horkheimer/Theodor W. Adorno, Dialektik der Aufklärung, Nijmegen 1944

51 Wolfgang Fritz Haug, das Ganze und das ganz Andere. Zur Kritik der reinen revolutionären Transzendenz, in: Antworten auf Herbert Marcuse, hrsg. v. Jürgen Habermas, Frankfurt/Main 1968

Wir nehmen an, daß die Kritiker hier meinen; „überschreiten, darüber hinausgehen“,

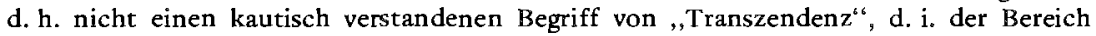
jenseits aller Erfahrung.

53 Wir weisen daraufhin, daß jene ,Arbeiten über den Zusammenhang von destruktiver Wissenschaftsverwendung und bürgerlich-kapitalistischer Gesellschaftsverfassung ", deren steigende Zahl die Kritiker begrüßen, und die sie ebenfalls zurückführen auf den Verlust an Fortschrittsoptimismus seit den Krisen der zwanziger Jahre, zum großen Teil von Autoren stammen, die sich selbst als Anhänger der Abbildtheorie verstehen; die Zeitschrift „Das Argument" war in dieser Angelegenheit übrigens nicht ganz untätig. Tatsächlich hat jenes Bediirfnis, das die Kritiker an materialistischer Erkenntnistheorie keimen sehen und das sie selbst teilen, mit den wissenschaftskritischen Bedürfnissen dieser analytischen Unternehmungen wenig zu tun. Letztere verdanken sich zumeist nicht einem Glaubensverlust am Fortschritt, sondern den Problemstellungen historisch-materialistischer Wissenschaft, und die Kritik bürgerlicher Wissenschaft ist deshalb auch nicht ein Krisenprodukt der zwanziger Jahre, sondern dem Marxismus von Anbeginn immanent. 
historischen Versuch, bürgerlich entfaltete Wissenschaft unter veränderten Produktionsverhältnissen nicht-destruktiv anzuwenden und ihre Möglichkeiten produktiv zu entwickeln, nur die Wiederkehr einer falschen, nämlich bürgerlichen Denk- und Erkenntnisform, und daher in den sozialistischen Staaten nur bürgerliche Gesellschaften sehen kann. Die „Klassiker" dagegen waren noch so naiv zu meinen, für die Möglichkeit des Sozialismus sei Voraussetzung, was die kapitalistische Gesellschaft an Produktionsmitteln geschaffen und an Produktivkraft entwickelt hat, und sie hielten es für eine „Dummheit..., nicht die kapitalistische Anwendurg der Maschinerie zu bekämpfen, sondern die Maschinerie selbst" (54). Wir wissen nun: ihre Naivität war „,heimlicher Positivismus“, und was sie für Dummheit hielten, ist wahrhaft revolutionäre Kritik.

\section{Erkenntnistheorie und materialistische Wissenschaft}

Marx und Engels haben bekanntlich nirgendwo positiv eine Erkenntnistheorie formuliert, hauptsächlich wohl deswegen, weil sie zeit ihres Lebens vollauf damit beschäftigt waren, die materialistische in ihrer wissenschaftlichen Arbeit implizit zu bewähren. Zwar machten sie sich bei gegebenem Anlass gelegentlich an die allgemein-philosophische Behandlung des „Erkenntnisproblems", wie z.B. Engels in seiner Polemik gegen Eugen Dühring, doch geschah dies dann nicht in der Absicht, eine besondere Erkenntnistheorie zu entwickeln, sondern vielmehr, um einer solchen entgegenzutreten. Wenn Engels sich gegen die Dühringsche Prinzipienlehre wendet, dann ,,transzendiert " er nicht die idealistischen Vorstellungen Dührings durch ausgeklügelte Metatheorie, sondern er unterläuft sie, indem er sie nämlich aus ihrer Verstiegenheit auf den Boden des Materialismus holt (55). Dabei geht er von der für jeden Materialisten selbstverständlichen Auffassung aus, daß die Welt objektiv, nämlich unabhängig vom wahrnehmenden und denkenden Menschen existiert und von diesem prinzipiell adäquat erkannt werden kann. Zu Engels' Zeit hatte diese Selbstverständlichkeit noch die völlig ungeteilte Zustimmung der übergroßen Mehrheit der bürgerlichen Naturwissenschaftler; weshalb auch für Marx und Engels die Durchsetzung des Materialismus in der Gescbicbtsauffassung - dort, wo die vom

Marx, Kapital I, MEW 23, S. 465. Marx führt in diesem Zusammenhang das ,Räsonnement des berühmten Gurgelschneiders Bill Sikes" an: ",Meine Herren Geschworenen, diesen Handlungsreisenden ist allerdings die Gurgel abgeschnitten worden. Diese Tatsache aber ist nicht meine Schuld, sie ist die Schuld des Messers. Sollen wir wegen solcher zeitweiligen Unannehmlichkeiten den Gebrauch des Messers abschaffen? Bedenken sie ja! Wo wäre Ackerbau und Handwerk ohne Messer? Ist es nicht ebenso heilbringend in der Chirugie wie gelehrt in der Anatomie? Dazu williger Gehilfe beim fröhlichen Mahl? Schaffen Sie das Messer ab - sie schleudern uns zurück in die tiefste Barbarei." " Die neuen kritischen Geschworenen von der radikalen Observanz lassen sich von Bill Sikes eher dahin überzeugen, daß er unschuldig ist, als daß sie es trotz seiner Verteidigung nicht für notwendig hielten, das Messer zu ,transzendieren“.

Vgl. Engels, Herrn Eugen Dührings Umwälzung der Wissenschaft, MEW 20, S. 32 ff. 
naturwissenschaftlich bestimmten ,Zeitgeist" bedrängten Bürger eine idealistiscbe Zuflucht suchten - an vorderster Stelle stand.

Was weder Marx noch Engels wissen konnten, war, daß künftige Generationen von Marxisten gezwungen sein würden, noch einmal die allgemeinsten Grundpositionen der materialistischen Wissenschaftsauffassung gegenüber einem neuen idealistischen bzw. agnostizistischen Angriff formulieren zu müssen. Die Notwendigkeit, positiv die Grundzüge einer marxistischen Erkenntnistheorie zu entwickeln, ergab sich aus der Tatsache, daß die neuen Angriffe sich jetzt nicht mehr frontal auf den bistorischen Materialismus, sondern, in listiger Umgehung des direkten Gegners, auf andere Bereiche der materialistischen Weltauffassung richtete. So ergab sich für die Marxisten eine Richtungsänderung im ideologischen Klassenkampf: Lenin wäre nie auf die Idee gekommen, ein derart ,banales“ Buch wie „Materialismus und Empiriokritizismus" ${ }^{6}$ zu schreiben, wenn ihn nicht der offene Agnostizismus der „Elementenlehre“ Ernst Machs und seiner sozialistischen Anhänger in Russland dazu genötigt hätte (56).

Dabei ging Lenin von der Einsicht aus, daß jeder Angriff auf die Einheit der materialistischen Weltauffassung als Gegner in letzter Instanz immer den bistorischen Materialismus im Visier hat: es geht stets um die Objektivität der Gesetzmäßigkeit des Geschichtsverlaufs und deren Erkennbarkeit. Wenn ein „Marxist ${ }^{\text {“ }}$ oder ein Kritiker des Marxismus die objektive Realität der materiellen Welt und ihre prinzipielle Erkennbarkeit durch den Menschen - und zwar ohne irgendwelche dazwischen geschobene kognitive „Prinzipien“ oder transzendentale Apperzeptioneń - an irgendeiner Stelle leugnet, dann verbirgt sich dahinter in der Regel ein Angriff auf diejenigen, die ihr politisches Handeln an den im historischen Materialismus allgemein formulierten Gesetzen des Geschichtsprozesses orientieren: nämlich die Vertreter des wissenschaftlichen Sozialismus. Das Hauptwerk des wissenschaftlichen Sozialismus, das „Kapital“, ist dann konsequenterweise keine gedankliche Reproduktion (und eben das ist wissenschaftliche Widerspiegelung) der objektiven Realität der kapitalistischen Gesellschaft samt ihrer historischen Tendenz, sondern bestenfalls das Produkt genialer Ordnungsprinzipien im Kopfe Karl Marx', verbunden mit moralisch dezidierter Kritik. Die von einem solchen Verständnis ausgehende Politik muß notwendigerweise subjektivistisch desorientiert sein, wenn nicht überhaupt in unmittelbar reaktionärer Absicht damit der Sozialismus wieder in eine Utopie rückverwandelt und Marx zum harmlosen Kulturkritiker gestempelt werden soll.

Die Widerspiegelungstheorie ist also zunächst einmal gar keine besondere „Theorie", sondern ein zusammenfassender Name für die allgemeine Grundlage der materialistischen Weltauffassung, die es gegen jeden Angriff des bürgerlichen Idealis-

Es handelt sich hier also nicht nur um ideologischen Klassenkampf, sondern vor allem darum, das Eindringen und die Ausbreitung bürgerlicher Auffassungen in der sozialistischen Bewegung zu verhindern. Aus dem gleichen Grunde schrieb Engels den AntiDühring. Marx' Analyse des Fetisch-Charakters der Ware im „Kapital“ war polemisch gerichtet gegen die Anhänger Proudhons unter den Sozialisten. 
mus oder Agnostizismus zu behaupten gilt - vor allem, wenn dieser Angriff im Gewande des „Marxismus" selber auftritt (57). Dies einmal klargestellt, kann man dann mit dem Namen ,Widerspiegelungstheorie" im engeren Sinne den Komplex all jener wissenschaftlichen Forschungen und Überlegungen bezeichnen, die sich - auf der Grundlage der objektiven Realität der Welt und ihrer prinzipiellen Erkennbarkeit - mit den Einzelbereichen und -bedingungen der menschlichen Erkenntnis von Natur und Gesellschaft befassen.

\section{Revolution der Erkenntnistheorie}

Wie der Feudale die Probleme der klassischen bürgerlichen Okonomie nicht verstünde, da ihm aus Unkenntnis des ,,Volksvorurteils von der menschlichen Gleichheit" (58) die Vorstellung, daß die menschliche Arbeit die Substanz des Wertes sei, absurd erschiene, so ist, analog, dem Bürger das Verständnis des marxistischen Widerspiegelungstheorems verwehrt. Da er, als ,homo faber" Kraftquell aller schöpferischen Tätigkeit sieht, bleibt ihm verschlossen, wo deren wirklicher Ursprung ist: in der Arbeit der Produzenten, die deshalb auch allein das praktische Kriterium der adäquaten oder verzerrten Widerspiegelung der objektiven Welt sein kann.

Allerdings hatte bekanntlich auch die bürgerliche Philosophie eine materialistische Auffassung von Erkenntnis als Widerspiegelung: die englischen Materialisten, vor allem Hobbes, dann die französischen Materialisten des 18. Jahrhunderts, sowie Feuerbach haben sie formuliert. Deren Vorstellungen waren jedoch, was die Struktur der Welt betraf, mechanistisch, und was den Prozeß der Widerspiegelung in der Erkenntnis anlangte, passivisch-anschauend. Sie beschränkten sich auf Naturerkenntnis und waren ahistorisch, da sie den gesellschaftlichen Zusammenhang einer materialistischen Erklärung nicht zugänglich machen, daher auch die gesellschaftlichen Bedingungen der Möglichkeit ihres eigenen Denkens nicht reflektieren konnten; in dieser Beziehung blieben sie dem Idealismus verhaftet. Eben diese Schwächen des frühbürgerlichen Materialismus werden nunmehr von spätbürgerlicher Kritik der marxistischen Erkenntnistheorie angelastet. Die Differenz zwischen der mechanistisch-passivischen Abbildtheorie und dem dialektischen Widerspiegelungstheorem wird treffsicher verfehlt. Denn unerträglich ist dem modernen Bürger beider Gemeinsames: der Materialismus.

57 „Die Dialektik der Geschichte ist derart, daß der theoretische Sieg des Marxismus seine Feinde zwingt, sich als Marxisten zu verkleiden" (Lenin, Werke 18, S. 578). Im übrigen ist dies ein in der Geschichte häufig vorkommendes Muster des ideologischen Abwehrkampfes: Thomas v. Aquin betrieb die ideologische Begründung der feudalklerikalen Reaktion mit den Mitteln des Aristotelismus, und die frechste Form der modernen bürgerlichen Gegenaufklärung, wie sie etwa Hayek und Popper betreiben, wird bezeichenderweise unter der Schutzmarke ,,kritischer Rationalismus" verkauft. 
Geschichtsmaterialistisch ist die Form des frühbürgerlichen mechanischen Materialismus unschwer in ihrer historisch-spezifischen Bedingtheit erkennbar: in ihm wurde der revolutionären Bürgerklasse die Entwicklung der gesellschaftlichen Produktivkräfte als ihre eigene historische Aufgabe bewußt, womit sich untrennbar - bei Francis Bacon ist dies klassisch zum Ausdruck gebracht - ihr Fortschrittsoptimismus verknüpfen konnte. Je weniger dieser Fortschrittsoptimismus sich aber bürgerlich aufrechterhalten ließ in einer gesellschaftlichen Wirklichkeit, die immer schärfer geprägt wurde von der Negation der bürgerlichen Klasse, dem Proletariat, die sie in Verfolgung ihrer historischen Aufgabe selbst hervorbrachte, desto mehr wich der Materialismus einer agnostischen Weltsicht.

Vom bürgerlichen Selbstverständnis, das als Einsicht in die eigene historische Mission aufs schönste eine materialistische Weltauffassung mit Fortschrittsoptimismus verbinden konnte, bleibt nur das bürgerliche Selbst: jenes Subjekt des cartesianischen ,cogito, ergo sum", das sich einst im Bewußtsein seiner objektiven historischen Berechtigung polemisch gegen die Autoritätsansprüche der scholastischen Theologie und deren unfruchtbare Logik richten konnte und den Materialismus bestens ergänzte, nun aber zur letzten konstituierenden Instanz aller Erkenntnis wird. Innerhalb einer der Bürgerklasse feindlich gegenüberstehenden gesellschaftlichen Realität wandelt sich dieses Subjekt zum ideologischen Substrat bürgerlichen Denkens. Allein und bei sich gelassen, geschichtlich hinfällig, lebt es als Subjektbewußtsein von der Fiktion seiner Autonomie. Diese Fiktion aber verträgt keinen Materialismus: der passivisch-anschauende ist $i b r$ nicht mehr möglich, der dialektische negiert sie. Die Verteidigungslinie des Subjektbewußtseins ist vorgezeichnet: der dialektische Materialismus, der es negiert, wird als der mechanische kritisiert, den es ,überwunden“" hat.

Diese Art offensiver Verteidigung ist innerhalb der spätbürgerlichen Ideologiebildung reich an Facetten. Sie kann sich konservativ gelassen oder positivistisch nüchtern geben, in jedem Fall beansprucht sie den Charakter modernster Wissenschaftlichkeit für sich. So auch die Kritiker, die nun allerdings eine besondere Version bürgerlicher Kritik der dialektisch-materialistischen Widerspiegelungstheorie repräsentieren. In bewährter Manier kritisieren auch sie diese als die mechanistische, passivische, ahistorische, naturalistische, wie sie das frühe Bürgertum hervorbrachte. Die üblichen Varianten moderner bürgerlicher Kritik sehen jedoch in der dialektischen Widerspiegelungstheorie allenfalls die Wiederkehr eines erkenntnistheoretischen Naivismus, der durch die Entwicklungen im Selbstreflexionsprozeß der Wissenschaften historisch längst ad acta gelegt ist. Die Kritiker dagegen wollen die marxistische Widerspiegelungstheorie entlarven (naiv ist sie ihnen natürlich ohnehin): als bloß marxistisch drapiert und im Kern bürgerlich. Damit annonciert sich diese Kritik als nicht-bürgerlich, als autbentiscb marxistisch (bürgerliche Kritik an der Widerspiegelungstheorie gibt es den Kritikern zufolge ja auch nicht, kann es in dieser Logik auch nicht geben).

Von dieser Kritik aus, die derart originär marxistisch ist, daß sie sogar ermöglicht, die dialektische Widerspiegelungstheorie als mechanistisch-bürgerlichen Scheinmarxismus zu enttarnen, ergibt sich dann auch klar, was eine ebenso wahr- 
haft marxistische Erkenntnistheorie leisten müßte. Prinzipiell hätte sie, wie wir schon wissen, alles hinter sich zu lassen, nämlich zu „transzendieren“, was herkömmliche bürgerliche Erkenntnistheorie einschließlich ihrer scheinmarxistischen Variante gedacht hat, und was aus ihr als Wissenschaft samt praktischen Folgen als Technik resultierte. Wie aber kann dieses Transzendieren vor sich gehen? Bei den Kritikern finden wir folgenden Hinweis auf das Programm einer konsequent marxistischen Erkenntnistheorie:

„Was eine marxistische Erkenntnistheorie von bürgerlicher Erkenntnistheorie unterscheidet bzw. unterscheiden muß, ist die Erklärung der Möglichkeit von objektiver (d.h. für alle Menschen gleichermaßen gültiger) Erkenntnis aus historisch-gesellschaftlichen Verhältnissen". (157; Hervorhebung die Verfasser)

Das klingt tatsächlich eminent marxistisch. Weiß man doch allgemein, daß der Marxismus alles auf ,gesellschaftliche Verhältnisse“ zuriickführt; mithin wäre er inkonsequent, täte er dies nicht auch mit der Erkenntnis. Nun wird andererseits kein marxistischer Widerspiegelungstheoretiker die Legitimität der Frage nach den historisch-gesellschaftlichen Bedingungen der Erkenntnis leugnen, im Gegenteil, schenkt man ihnen Glauben (die Kritiker tun dies freilich nicht), so ist innen das Problem sogar besonders wichtig. Wo also liegt dann der Unterschied zwischen diesem Programm der Kritiker und den Positionen der dialektisch-materialistischen Widerspiegelungstheorie?

Wenn wir zur Klärung dieser Frage das Programm der Kritiker, die Möglichkeit von Erkenntnis aus historisch-gesellschaftlichen Verhältnissen zu erklären, näher beleuchten, so kommt uns dabei der Umstand zu Hilfe, daß es erst kürzlich nicht nur verkündet, sondern auch absolviert vorgelegt wurde: von Alfred SohnRethel. Er erklärt die Möglichkeit von Erkenntnis aus historisch-gesellschaftlichen Verhältnissen, und wir können daher an ihm die Implikationen dieses Programms sehr gut verfolgen (59). In der Einleitung zu seinem Buch „Geistige und körperliche Arbeit" heißt es:

„Dem Individuum selbst allerdings, zumal dem modernen, erscheint sein vergesellschaftetes Denken ... als Leistung seines, dem Ursprung nach zwar mysteriösen (Gott gegebenen und doch Gott verneinenden), der Logik nach aber autonomen und ihm ureigenen, ego cogitans'. Unserer Erklärung gemäß sind jedoch diese Kategorien gesellschaftlich vorgeformt und daher so,

59 Wir müssen betonen, daß wir keineswegs unterstellen wollen, die Kritiker verstünden sich als Schüler Sohn-Rethels. Man hat zwar den Eindruck, daß sie manches von ihm bezogen haben, ohne dies zu kennzeichnen. aber ein solcher direkter Zusammenhang würde uns nicht interessieren. Es geht uns um die Struktur des Programms und des Bewußtseins, das sich darin äußert, nicht um irgendwelche intellektuelle Gen ılogien. Allerdings ist bemerkenswert, daß die Kritiker Sohn-Rethel nur in einer Fußnote erwähnen (166, Anm. 27), die sie benutzen, eine Rezension von Frigga Haug zu Sohn-Rethels opus maximum (vgl. Anm. 60) als ,, an Unverständnis nicht zu überbietende Stellungnahme" zu disqualifizieren, ohne auch nur den Anschein irgendeiner Begründung. dafür vorzubringen (Vgl. F. Haug, in: Das Argument, 65, 1971, S. $313 \mathrm{ff}$.) 
wie sie ans Individuum gelangen, ihm in fertiger (aber darum noch lange nicht identifizierbarer) Form gegeben, also tatsächlich Kategorien , a priori‘ und auch für alle Individuen dieser Gesellschaft natürlich identisch dieselben. Kant wußte, daß sie vorgeformt sind, aber er verlegte den Vorformungsprozeß ins Bewußtsein als eine sowohl zeitlich wie örtlich unlokalisierbare, phantasmagorische, transzendentale Synthesis'. In Wirklichkeit ist die vorformende Synthesis der abstrakten Kategorien ein geschichtlicher Prozeß und nur bestimmten, klar definierbaren Gesellschaftsformationen eigen. Am Gelde, genauer an seiner gesellschaftlich-synthetischen Funktion, haften Züge von unverkennbarer Portraitähnlichkeit ... mit dem ,Transzendentalsubjekt', zumal der Formcharakter, vermöge dessen das Geld durch alle Verschiedenheit der Währungen hindurch universell nur eines sein kann" (60).

Sohn-Rethel befindet sich also im Gegensatz zu Kant. Mit ihm teilt er zwar das Wissen, daß die Kategorien des erkennenden Verstandes, ,vorgeformt " sind, nicht aber die Antwort auf die Frage: wovon? Diese Frage beantworten beide ganz unterschiedlich. Gültige Erkenntnis, wie sie sich in den Naturwissenschaften und ihren praktischen Erfolgen zeigt, findet ihre Konstitutionsbedingungen laut Kant in der ,transzendentalen Einheit der reinen Apperzeption“. Diese Antwort Kants deren nähere Präzisierung im vorliegenden Zusammenhang nicht vonnöten ist verwirft Sohn-Rethel als ,phantasmagorisch“. Der ,transzendentalen Synthesis“ als Vorformungs-Instanz der Kategorien und der begrifflichen Verarbeitung des sinnlich Erfahrenen, die er als idealistisch aufdeckt, setzt Sohn-Rethel, ganz im Sinne des Kritiker-Programms, die ,gesellschaftliche Synthesis“ als Vorformungs-Instanz gegenüber.

Die besonderen „historisch-gesellschaftlichen Verhältnisse“ nun, aus denen Sohn-Rethel die Möglichkeit intersubjektiver Gültigkeit von synthetischen Urteilen a priori, der ,abstrakten Kategorien“" also, ableitet, sind die der warenproduzierenden Gesellschaft und ihrer "gesellschaftlichen Synthesis“, des Warentauschs bzw. mit ihm verbundenen „Realabstraktion“. Diese „prägt“ (ganz wörtlich mit Hilfe des historisch auftretenden Münzgeldes) die Denkform des abstrakten Denkens - zuerst in der griechischen Antike, dann wieder neu in den oberitalienischen Städten, (61) als Denkform einer besonderen Form der Ausbeutergesellschaft, nämlich der ,wechselseitigen Aneignungsgesellschaft"“, d.h. der Tauschgesellschaft, zu deren Typus auch der Kapitalismus gehört (62). Dies soll die materialistische Antwort auf Kants Frage nach der Vorformungs-Instanz der „abstrakten Kategorien“, also nach dem Ort der Konstitution von Erkenntnis sein.

Ist es eine materialistische Antwort? Wir sehen zunächst, daß Kants überbistorische und einem menschheitlich gleichen Bewußtsein als Funktion inhärente

Alfred Sohn-Rethel, Geistige und körperliche Arbeit. Zur Theorie der gesellschaftlichen Synthesis, Frankfurt/Main 1972, S. $21 \mathrm{ff}$.

61 Vgl. Sohn-Rethel, a.a.O., S. $137-149$

62 Die Widergabe der Theorien Sohn-Rethels wurde hier auf das knappste Maß dessen beschränkt, was für die Zwecke unserer Argumentation nötig ist. Unsere kritischen Anmerkungen, in denen Sohn-Rethel nur als Beispiel für einen bestimmten Denktypus fungiert, implizieren keine wirklich gediegene Sohn-Rethel-Kritik. Eine solche steht von marxistischer Seite noch aus, wäre aber, wie wir meinen, verlohnend, weil sich bei Sohn-Rethel, wenn auch verquer, bedenkenswerte Einsichten finden. 
Synthesis in der Tat ersetzt ist durch eine bistoriscbe und einer spezifischen Form von Gesellscbaft als Struktur inhärente Synthesis. Wir sehen weiter, wie die „,abstrakten Kategorien“ als derart präzise Prägungen historisch-gesellschaftlicher Verhältnisse verdeutlicht werden, daß man den Zusammenhang zwischen dieser Gesellschaft und diesem Denken ohne weiteres sogar als Widerspiegelung im Wortsinne (,unverkennbare Portraitähnlichkeit“) bezeichnen könnte. Wir sehen ferner, daß Sohn-Rethel eindeutig Partei ergreift für die Ausgebeuteten und also einen klaren Klassenstandpunkt einnimmt: In der Kant'schen Gegenüberstellung von „Ding an sich“, dem unerkennbaren Wesen der Realität, und sinnlicher Erfahrung, die der Erkenntnis nur das Material amorpher Empfindungen liefern kann, entschlüsselt er die historisch-gesellschaftliche Trennung von Kopf- und Handarbeit.

All dies scheint dafür zu sprechen, daß wir mit Sohn-Rethels Lehre die zentralen Elemente einer wahrhaft materialistischen, originär marxistischen Erkenntnistheorie vor uns haben. Ein weiterer Vorteil dieser Position kommt hinzu: sie versieht den, der sich zu ihr bekennt, mit präzisen Urteilskriterien hinsichtlich der Bürgerlichkeit von Gesellschaftsstrukturen. Praktisch politisch kann man nun als Regel nehmen, daß dort, wo Warenproduktion, Warentausch und Geld, also auch Trennung von Kopf- und Handarbeit, also auch das Denken in abstrakten Kategorien mit all seinen Folgen als Wissenschaft und Technik das gesellschaftliche Leben (noch) bestimmt, die Synthesis der bürgerlichen Gesellschaft gegeben ist, in welcher historischen Spezifizierung auch immer.

Somit reicht die Sohn-Rethel'sche authentisch marxistische Erkenntnislehre hin, nicht nur das Programm der Kritiker zu erfüllen, sondern auch deren politische Befürchtungen zu fundieren. Was die Kritiker in ihrer Analyse der marxistischen Widerspiegelungstheorie an Bürgerlichem aufdecken, ist dann als Reflex einer nach wie vor bürgerlichen gesellschaftlichen Synthesis keineswegs verwunderlich; wie sie, wendet man die Kriterien Sohn-Rethels an, auch die Länder prägt, in den die marxistische Widerspiegelungstheorie die offizielle Auffassung in Fragen der Erkenntnis darstellt. Hier konvergieren nicht nur Ost und West im Bürgerlichen, sondern auch Sohn-Rethel und die Kritiker im anti-bürgerlichen Programm: es kommt darauf an, den „Marxismus als Denkstandpunkt“ (Sohn-Rethel) zu retten:

\begin{abstract}
„Zur Schaffung des Sozialismus wird verlangt, daß es der Gesellschaft gelingt, sich die moderne Entwicklung von Naturwissenschaft und Technologie zu subsumieren. Wenn die naturwissenschaftlichen Denkformen und der technologische Aspekt der Produktivkräfte sich aber der geschichts-materialistischen Betrachtungsweise wesensmäßig entziehen, so ist eine solche Subsumption unmöglich. Dann geht die heutige Menschheit nicht dem Sozialismus, sondern der Technokratie entgegen, einer Zukunft also, in der nicht die Gesellschaft über die Technik, sondern die Technik über die Gesellschaft herrscht. Wenn es dem Marxismus nicht gelingt, der zeitlosen Wahrheitstheorie der herrschenden naturwissenschaftlichen Erkenntnislehren den Boden zu entziehen, dann ist die Abdankung des Marxismus als Denkstandpunkt eine bloße Frage der Zeit. Und dies ist nicht nur mit Geltung für die westliche Welt gesagt, wo das technokratische Denken sich auf den Positivismus stützt, es gilt kaum weniger für manche sozialistische Länder, in denen der Technokratic im Namen des, dialektischen Materialismus gehuldigt wird" (63).
\end{abstract}


Ist es also eine materialistische Antwort? Natürlich handelt es sich bei Sohn-Rethels Auffassungen keineswegs um materialistische Erkenntnistheorie, so anregend manche seiner Gedanken auch für diese sein mögen. Diese negative Bestimmung ergibt sich nicht etwa aus der eben zitierten konvergenztheoretischen Begründung eines sogenannten "Anti-Revisionismus"; diese ist vielmehr selbst erst ermöglicht durch den nicht-materialistischen Charakter des Programms, Erkenntnis ,aus historisch-gesellschaftlichen Verhältnissen“ erklären zu wollen. Dieses Programm der Kritiker führt nämlich - und das kann auch an Sohn-Rethel deutlich werden nicht etwa zu einer historisch-materialistischen, sondern zu einer soziologistiscben Theorie der Erkenntnis.

Die Wirklichkeit selbst bleibt nach wie vor „Ding an sich“", also unerkennbar; erkannt werden nur, und daher sind Naturwissenschaft und Technik möglich, die Erscbeinungen der absoluten Realität. Insoweit reproduziert sich der Kant'sche Idealismus bei Sohn-Rethel vollkommen. Das Verhältnis von Subjekt und Objekt der Erkenntnis ist grundsätzlich das gleiche wie bei Kant: sie stehen sich ohne Vermittlung durch Praxis abstrakt gegenüber. Beim Auswechseln der die Möglichkeit von Erkenntnis der Erscheinungswelt konstituierenden Vorformungs-Instanzen, der ,transzendentalen Synthesis“ durch die ,,gesellschaftliche Synthesis" der warenproduzierenden Tauschgesellschaft, wird jedoch die idealistische Antwort auf die Frage nach den Konstitutionsbedingungen von Erkenntnis nicht etwa durch eine materialistische ersetzt, Erkenntnis wird vielmehr radikal historisiert und soziologisiert. Daraus resultiert nun tatsächlich ein uferloser Relativismus in der Erkenntnistheorie, den Kant nicht kannte: seine „transzendentale Synthesis“ implizierte, in wenn auch noch so ,phantasmagorischer“, idealistisch verkehrter Form im „Transzendentalsubjekt" die menscbbeitlicbe Identität der Erkenntnisfäbigkeit, die sich, dialektisch-materialistisch zu sich gebracht, als Produktion der dialektischen Bewegung der Materie erweist; so steht der objektive Idealismus Kants, der frühbürgerlichem Fortschrittsoptimismus noch kommensurabel war, dem Materialismus weit näher als der Soziologismus Sohn-Rethels, der tatsächlich eine gesellschaftskritisch gewendete Version spätbürgerlichen Agnostizismus darstellt.

Dem entspricht, daß Sohn-Rethel eine nun wirklich passivische Form von Widerspiegelung entwirft. Es handelt sich ja nicht um den dialektischen Widerspiegelungsprozeß zwischen objektiver Realität und erkennendem Subjekt, dessen Medium gesellschaftliche Praxis ist, sondern um eine direkte und einseitige „Prägung“ des Bewußtseins durch die gesellschaftliche Synthesis: die „Realabstraktion“ der Tauschgesellschaft spiegelt sich als System abstrakter Kategorien im Bewußtsein. Eine strukturell ähnliche, in der Begründung allerdings differente, passivische Widerspiegelungstheorie hat übrigens bereits Franz Borkenau entworfen (64). Und Borkenau sieht allerdings die soziale Basis des ",mechanistischen Denkens" im unmittelbaren Arbeitsprozeß, während Sohn-Rethel den ,gesellschaftlichen Nexus" des Warentauschs als diese Basis diagnostiziert. Die Fragestellung ist jedoch grundsätzlich dieselbe. 
was schließlich den klaren Klassenstandpunkt anlangt, der Sohn-Rethels Theorie auszeichnet, so erweist er sich bei näherem Hinsehen als bloß moralisch eingenommen, denn es handelt sich gar nicht um eine wirklich bistoriscb angelegte Theorie: die klassenspezifisch bestimmten Differenzen der Gesellschaften, in denen abstrak tes Denken entwickelt wurde, werden eingeschmolzen in einen allgemeinen Typus - den der „Aneignungsgesellschaft“", der gekennzeichnet ist durch „Scheidung von körperlicher und geistiger Arbeit" (65). Solche historistische Typologie, die dem grundsätzlichen Soziologismus entspricht, abstrahiert gerade vom historisch bestimmten Inhalt der Klassenauseinandersetzungen unserer Epoche.

Es versteht sich, daß wir die Kritiker nun nicht etwa auf die Details der Lehre Sohn-Rethels festlegen wollen und können. Wir behaùpten aber, daß dessen Auffassungen eine legitime Möglichkeit sind, ihr Programm auszufüllen. Wir wollten sichtbar machen, was aus einer bloßen Programm-Formulierung nicht unmittelbar evident wird, und haben uns dabei der Auffassungen Sohn-Rethels bedient. Unsere allgemeine These ist, daß die Absicht, die Möglichkeit von objektiver Erkenntnis aus historisch-gesellschaftlichen Verhältnissen zu erklären, soweit sie sich als Alternative zur dialektisch-materialistischen Widerspiegelungstheorie versteht, grundsätzlich nicht $z u$ einer materialistiscben Erkenntnistbeorie fübren kann, sondern in einem agnostiscb geprägten Soziologismus enden muß, der ironischerweise gerade das impliziert, was die Kritiker der dialektischen Widerspiegelungstheorie meinen vorwerfen zu können, nämlich haltlosen Relativismus.

Nicht, daß Gescbichte in Fragen der Erkenntnistheorie für Materialisten keine Rolle spielte; eben diese Auffassung möchten, wie wir gesehen haben, die Kritiker der Widerspiegelungstheorie unterschieben. Materialistische Erkenntnistheorie geht vielmehr davon aus, daß die Bedingungen der Realisierung von Erkenntnis historisch-gesellschaftlich sind (und insoweit bergen die Gedanken Sohn-Rethels zweifellos interessante Aspekte). Sie weiß aber auch, daß diese Realisierung materielle Bedingungen zur Voraussetzung hat, die sie überhaupt erst ermöglichen (und die Frage nach diesen Voraussetzungen ist ihr primäres Thema). Diese Bedingungen sind Resultate der Naturgeschichte und ihrer Dialektik, sind nicht erst von der Menschheit gesellschaftlich-historisch produziert worden, so sehr die Menschheitsgeschichte auch auf die organisch-sinnliche Erkenntnisfähigkeit modifizierend einwirkt. Eben diese Voraussetzung wollen die Kritiker annullieren. Soll die Möglichkeit von Erkenntnis selbst, und nicht erst die Chancen der Realisierung von

„,Die Kategorie ,arbeitsteilige Gesellschaft überhaupt ist höher, allgemeiner, als die ,kapitalistische Gesellschaft", aber nicht wesentlicher, sondern unwesentlicher, sagt weniger uber das Leben der Menschen und das, was sie bedroht, ohne daß doch darum eine logisch niedrigere Kategorie ,Urbanismus" mehr darüber besagte. Weder nach oben noch nach unten entsprechen soziologische Abstraktionsniveaus einfach dem gesellschaftlichen Erkenntniswert" (Th. W. Adorno, Soziologie und empirische Forschung, in: Horkheimer/Adorno, Soziologica II, Frankfurt/Main 1962, S、206). -- In diesem Sinne wäre noch hinzuzufügen, daß sich Sohn-Rethel, was die Prinzipien seiner sozialwissenschaftlichen Begriffsbildung betrifft, tatsächlich etwa Parsons näher ist als Marx. 
Erkenntnisfähigkeit, aus ,historisch-gesellschaftlichen Verhältnissen“ erklärt werden, so ist zwischen materiellen Voraussetzungen und historisch-gesellschaftlichen Bedingungen der Realisierung von Erkenntnis nicht zu unterscheiden. Die bürgerliche Gesellschaft kann dann nicht als menschheitliches Entwicklungsstadium begriffen werden, in dem die aus der Naturdialektik resultierende höchste Form des Widerspiegelungsvorganges als menschliche Erkenntnis bestimmte, allerdings durch den Klassen- und Herrschaftscharakter dieser Gesellschaft beschränkte, Realisierungschancen erhält - umgekehrt: die in dieser bürgerlichen Gesellschaft ausgebildete Wissenschaft wird zu einer ihr historisch-spezifischen Form von Ideologie, die es zu ,transzendieren" gilt.

Dies aber ist nun der springende Punkt, auf den es dem spätbürgerlichen Subjektbewußtsein ankommen muß. Der Mensch wird in dieser Perspektive zum ausscbließlich gesellschaftlichen Wesen. Keine Naturgeschichte hat ihn hervorgebracht, sondern einzig er sich selber, sowie die Gesellschaft, die er aber auch geschaffen hat. So gibt es nur Menschheitsgeschichte, die (leider) die Geschichte menschheitlicher Verblendungen ist. Warum der historische Materialismus historischer Materialismus heißt, wird apokryph: unterstellt wird, das strikte Insistieren auf das Gesellschaftliche und das Historische bei der Bildung menschlicher Bewußtseinsformen sei an sich scbon materialistisch. Vom historischen Materialismus bleibt nur die Historie, soziologisch betrachtet. Offensiv wird diese Auflösung von Naturdialektik in Menschheitsgeschichte derart gewendet, daß der dialektisch-materialistischen Widerspiegelungstheorie vorgeworfen wird, Menschheitsgeschichte in Naturgeschichte aufzulösen: es gebe für sie nur Naturgeschichte (vgl. oben I,3).

Durch Radikalisierung des Geschichtlichen wird so der historische Materialismus überrundet, Geschichtsmaterialismus durch nichts als Geschichte ausgestochen. Darin, in nichts als Geschichte, rettet die linke Variante des spätbürgerlichen Subjektbewußtseins die Fiktion seiner Autonomie: sie ist Ideologie im strikten Sinne des falschen Bewußtseins. Durch die pure Ambition der intellektuellen Revolte bietet es der gesellschaftlichen Synthesis der „Realabstraktion“ Paroli und bleibt getrost bei sich zu Hause. Gleichzeitig schwingt es sich zur kritischen Instanz auf, die die Maßstäbe für das Urteil über gesellschaftliche Verhältnisse aus seinem schöpferischen Akt des Transzendierens bezieht. Denn diese Verhältnisse verdanken sich ihm ja nicht etwa - materialistisch-objektiven Gesetzmäßigkeiten, sondern letztlich idealistisch-menschheitlichen Verblendungszusammenhängen. Eine Phänomenologie des spätbürgerlichen Linksradikalismus, in seiner modischen Fasson als ,Anti-Revisionismus" (der Begriff enthält in sich schon alles, was wir erklären wollten), hätte die Fülle der besonderen Formen dieses Bewußtseins aus der skizzierten gemeinsamen Struktur zu erklären. Stets aber realisiert sich diese intellektuelle Disposition in platten Identifikationen. Aus deren Verschiedenheit ergeben sich die mitunter erbitterten Fehden zwischen den Trägern dieses strukturell gleichen links-spätbürgerlichen Bewußtseins. Eines aber ist die Bedingung für Identifikation: das, womit man sich identifiziert, muß als das Ganz Andere, als das von den Verblendungen der Geschichte Gereinigte erscheinen können. Es kann sich auf Realitäten beziehen, sagen wir mal: auf China (so u. a. auch bei Sohn-Rethel); 
dann muß diese Realität aber so fern und so exotisch sein, daß ihre Widersprüche nicht scharf ins Bewußtsein treten und das Identifikationsbedürfnis stören müssen. Schwerer überprüfbar ist die Fixierung, wenn sie sich aif eine ungedachte Utopie bezieht, deren Konturen nicht auszumachen sind und die nur den Fluchtund Beziehungspunkt radikaler Kritik bildet, wie bei den Kritikern. Wie immer: von diesem Ganz Anderen aus, in dem es sein Wolkenkuckucksheim hat, kann dieses Bewußtsein marxistische Erkenntnistheorie verkünden als Revolution des Umdenkens.

\section{Helmut Fleischer Sozialphilosophische Studien \\ Kritik der marxistisch-leninistischen Schulphilosophie}

Es gibt einen offiziellen Marxismus, der zur ideologischen Fassade der politischen Entmündigung der Arbeiterklasse geworden ist. Ihre Befreiung ist demzufolge kodifiziert in einem naturgesetzlichen Entwicklungsprogramm, das eine eigens dazu ernannte Elite hütet, auslegt und durchsetzt.

Es gibt aber auch einen subversiven Marxismus, der die Geschichte nach den Bedingungen der Abschaffung von Herrschaft untersucht, und dies ist unlöslich an die massenhafte Selbstverwirklichung der Arbeiter geknüpft.

Diese marxistische Subversion ist keine unbefleckte Empfängnis durch den kritischen Geist, sondern die widersprüchliche Formulierung proletarischer Emanzipationsversuche, denen die einzelnen Theoretiker halb entsprechen, halb entfliehen in Prinzipienidealismus, Mystik und realpolitischen Opportunismus. Das Werk von Lukasc, Korsch, Bloch ist von diesen Risiken einer theoretischen Formulierung der emanzipativen Interessen der Arbeiter durchzogen; trotzdem sind ihre Versuche unentbehrlich, um überhaupt einen Begriff von den Zielen zu bekommen, an Hand deren man die Wege beurteilen könnte.

Der Rang von Fleischers Werk liegt in einer schlüsssigen Interprotation der Handlungsantriebe der historischen Subjekte die sich kritisch gegen den verdinglichten Geschichtsschematismus der sowjetischen Schulphilosophie kehrt. Daß hior keine Gedankenverwirrung vortiegt sondern materielle Gewalt zur Theorie geworden ist, legt Fleischer in der vorliegenden Auf satzsammlung dar. Die Deformation der Theoriebildung, gemessen an den marxschen Zwecken, erweist sich als sehr praktisch zur Steuerung des Gesellschaftssystems der DDR und UdSSR. Fleischer interpretiert nicht allein diesen Zustand, sondern belebt die marx'schen Kriterien, um ihn zu verändern.

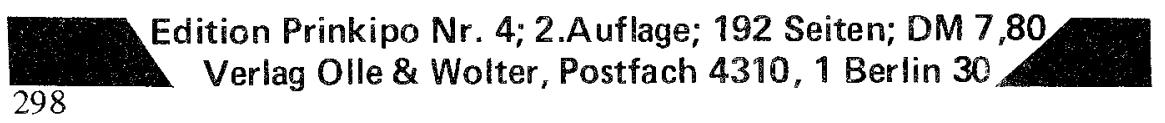

